\title{
Semantic Contextual Cuing and Visual Attention
}

\begin{abstract}
Annabelle Goujon
Centre National de la Recherche Scientifique, University of

Provence, and Institut National de Recherche sur les Transports

et leur Sécurité

Evelyne Marmèche

Centre National de la Recherche Scientifique and University of Provence

\author{
André Didierjean \\ University of Franche-Comté
}

Since M. M. Chun and Y. Jiang's (1998) original study, a large body of research based on the contextual cuing paradigm has shown that the visuocognitive system is capable of capturing certain regularities in the environment in an implicit way. The present study investigated whether regularities based on the semantic category membership of the context can be learned implicitly and whether that learning depends on attention. The contextual cuing paradigm was used with lexical displays in which the semantic category of the contextual words either did or did not predict the target location. Experiments 1 and 2 revealed that implicit contextual cuing effects can be extended to semantic category regularities. Experiments 3 and 4 indicated an implicit contextual cuing effect when the predictive context appeared in an attended color but not when the predictive context appeared in an ignored color. However, when the previously ignored context suddenly became attended, it immediately facilitated performance. In contrast, when the previously attended context suddenly became ignored, no benefit was observed. Results suggest that the expression of implicit semantic knowledge depends on attention but that latent learning can nevertheless take place outside the attentional field.
\end{abstract}

Keywords: contextual cuing, implicit learning, semantic, selective attention

A large body of research based on the contextual cuing paradigm has shown how implicit knowledge of the regularities in the environment can direct the observer's attention within a scene (Chun \& Jiang, 1998). The first goal of the present study was to determine whether such implicit learning can occur when the regularities concern the semantic category of the context. The second goal was to look into how implicit learning of semantic regularities might be modulated by attention.

Current research on the perception of natural scenes, particularly work on change detection, has indicated drastic visual memory limitations from one view to the next (for a review, see Simons \& Rensink, 2005). Change blindness phenomena, sometimes even dramatic ones, have led some authors to suggest that contrary to our phenomenology, according to which conscious representations are copies of the real world, such representations are rather sparse and volatile (e.g., Irwin \& Andrews, 1996; Rensink, 2000; but also Hollingworth \& Henderson, 2002). However, our attention is nevertheless directed rapidly and efficiently toward elements in the

Annabelle Goujon, Centre National de la Recherche Scientifique, Marseille, France; Department of Psychology, University of Provence, Marseille, France; and Institut National de Recherche sur les Transports et leur Sécurité, Salon de Provence, France. André Didierjean, Department of Psychology, University of Franche-Comté, Besançon, France. Evelyne Marmèche, Centre National de la Recherche Scientifique and Department of Psychology, University of Provence.

Correspondence concerning this article should be addressed to Annabelle Goujon, who now is at the School of Philosophy, Psychology, and Language Sciences, University of Edinburgh, 7 George Square, Edinburgh, UK EH8 9JZ. E-mail: annabelle.goujon@ed.ac.uk scene that are relevant to our goals. During driving, for example, attentional processes select the necessary information for the driving task from the rich and ever-changing visual environment. What factors are responsible for this adaptability, knowing that very little of this information is available to awareness at a given instant? It is widely accepted that preexisting knowledge about the current scene and situation plays a determining role in directing attention. Knowledge about environmental regularities is thought to be stored in long-term memory in the form of a scene schema, which describes objects and events likely to be present in the scene, along with their locations with respect to each other (Mandler \& Ritchey, 1977; Potter, 1975). The early activation of this knowledge during the analysis of a scene would account for the effectiveness of visual processing, not only by facilitating scene interpretation and identification of the objects they contain (Biederman, Mezzanotte, \& Rabinowitz, 1982) but also by facilitating scene exploration (Friedman, 1979; Henderson, Weeks, \& Hollingworth, 1999). A tempting hypothesis is that some of the knowledge in scene schemas is acquired and used implicitly. Implicit learning refers to an adaptation process through which the behavior of an individual becomes sensitive to a structure in an incidental manner, in such a way that the individual is not able to verbally report or even consciously access the resulting knowledge (for reviews, Reber, 1989; Seger, 1994). Such knowledge could affect the unfolding of visuocognitive or visuomotor operations. In this framework, two fundamental questions remain unanswered. The first concerns the nature of implicit knowledge: Is it simply a specific and especially perceptual kind of knowledge, or could it be conceptual in nature, that is, based on a semantic categorization of the elements present in the scene? The second question concerns the role of selective 
attention in the implicit learning of regularities in the environment: Is selective attention to contextual elements required for the learning of semantic regularities to take place?

To address these issues in the visual perception framework, we used the contextual cuing paradigm developed by Chun and Jiang (1998). This paradigm generally involves two tasks: a visual search task and a verbalization-then-recognition task. In a classical visual search task, participants are asked to look for a particular target (e.g., a $T$ pointing to the right or left) among a number of distractors that form the context of the target (e.g., a set of $L s$ pointing in different directions). The participants are exposed to many blocks of trials. Half of the trials in a given block are predictive, and the other half are nonpredictive. In a predictive trial, regularity in the context (e.g., the layout of the distractors) predicts the target's location; in a nonpredictive trial, the context is random and does not predict the target's location. The existence of a contextual cuing effect is reflected by increasingly shorter target detection times in the predictive condition than in the nonpredictive condition. Learning of the association between the context and the target is thought to facilitate the visual search by automatically directing attention toward the target location (Chun \& Jiang, 1998; Peterson \& Kramer, 2001). It often turns out that contextual cuing effects are obtained without awareness of the regularities that nonetheless facilitated the search. Participants are usually incapable of stating the regularities and cannot even use them to differentiate between predictive and nonpredictive trials in a recognition task performed immediately after the search task (Chun \& Jiang, 1998, 2003). Moreover, Chun and Jiang (2003) showed that search performance and explicit memory performance in spatial contextual cuing were not improved by explicit instructions requesting participants to actively encode contextual regularities. The contextual cuing paradigm thus constitutes a potentially relevant tool for investigating the implicit learning mechanisms involved in visual perception.

In most studies, implicit contextual cuing effects have been obtained from displays in which the regularities manipulated were specific features of the context, such as the spatial layout of the contextual elements (e.g., Jiang, Song, \& Rigas, 2005; Jiang \& Wagner, 2004; Ono, Jiang, \& Kawahara, 2005), their shape (Chun \& Jiang, 1999; Endo \& Takeda, 2004), or their temporal relationship (Chun \& Jiang, 1999; Olson \& Chun, 2001). Contextual cuing effects have also been obtained using real-world scenes containing semantic cues (e.g., Brockmole, Castelhano, \& Henderson, 2006; Brockmole \& Henderson, 2006b). Brockmole and Henderson (2006a) showed that when natural scenes were systematically repeated, attention was guided first by the global identity of the scene and second by the specific visual features of objects present in the picture. However, in this case, the observed learning effects were explicit ones. Contextual cuing effects based on the semantic categorical properties of the context have also been observed in less natural environments (Goujon, Didierjean, \& Marmèche, 2007). Goujon et al. (2007) showed that the semantic category (even vs. odd) of numerical contexts could act as a cue to the location of a particular target number. However, this time the observed contextual cuing effects were implicit. Indeed, after the search task, the participants did not mention the evenness-oddness property and were unable to use this property on a recognition task. The first aim of the present study was to extend these effects to lexical semantic categorical properties. The second aim was to find out whether implicit learning and retrieval of semantic category regularities necessarily require selective attention in a contextual cuing task.

The first question concerns the depth (perceptual or conceptual) of unconscious cognitive processing. In priming research, the existence of unconscious semantic processing of masked primes has been the subject of numerous debates. Indeed, because in many studies the same items are used as primes and targets (e.g., Marcel, 1983), the observed effects can be interpreted in two ways: in terms of priming based on the semantic properties of the words or in terms of repetition priming based simply on their specific form (Abrams \& Greenwald, 2000; Damian, 2001). Recently, several studies seem to show that priming can, in fact, be based on an abstract kind of prime processing and not only on the mere processing of forms (Klauer, Eder, Greenwald, \& Abrams, 2007; Naccache \& Dehaene, 2001). Of interest, a comparable and equally heated theoretical and methodological debate arose in the area of implicit learning (for a review, see Cleeremans, Destrebecqz, \& Boyer, 1998). Here again, the controversy lies in the multiplicity of interpretations proposed to account for the results. When implicit learning effects are observed, are they simply due to the extraction of specific features or can abstract properties be involved? This question is still widely debated in the literature on implicit learning, especially in studies on artificial grammar learning (for a review, see Pothos, 2007). According to some authors (e.g., Knowlton \& Squire, 1996; Reber, 1989), people are capable of incidentally and automatically acquiring tacit knowledge about an abstract unconscious representation of the structure contained in the information presented, whereas others maintain that implicit learning is based only on extraction of specific perceptual properties of the material (e.g., Perruchet \& Pacteau, 1990; Perruchet Vinter, Pacteau, \& Gallego, 2002; Vokey \& Brooks, 1994).

The second question raised in the present study concerns the role played by selective attention in implicit learning. This, too, is a highly debated issue. Implicit learning is generally likened to automatic processing, which requires little or no attention. In this view, it is thought to be an "unselective and passive aggregation of information about the co-occurrence of environmental events and features" (Hayes \& Broadbent, 1988, p. 251). Arguing against this point of view, more and more experimental results are suggesting that implicit learning is modulated by selective attention (for reviews, see Chun \& Turk-Brown, 2007; Perruchet \& Pacton, 2006). For example, implicit visual statistical learning seems to be modulated by selective attention in tasks where forms covary in time (Turk-Browne, Jungé, \& Scholl, 2005), in spatial contextual cuing tasks (Jiang \& Chun, 2001), or in serial reaction-time (RT) tasks (e.g., Remillard, 2003). However, recent findings have revealed some interesting phenomena. Although the expression of implicit learning (manifested by lower detection times in repetition conditions than in random conditions) requires selective attention, latent learning that is independent of attention directed at the manipulated visual regularities may nevertheless occur (Frensch, Lin, \& Buchner, 1998; Frensch, Wenke, \& Rünger, 1999; Jiang \& Leung, 2005). Indeed, Jiang and Leung (2005) showed using the contextual cuing paradigm that an ignored repeated context did not facilitate performance (see also Jiang \& Chun, 2001), but when that context suddenly became attended, performance immediately improved. These results suggest that although the expression of contextual cuing depends on the repeated contexts being attended, 
latent learning can nevertheless occur on an ignored context. It is important to note, however, that these findings pertain only to the implicit learning of nonsemantic perceptual regularities.

In the study presented here, four experiments were conducted using the contextual cuing paradigm with word displays. Each experiment included a search task to test for learning of contextual regularities, followed by a verbalization-then-recognition task aimed at determining the implicit or explicit nature of the learning that potentially took place in the search task. Experiments 1 and 2 tested whether implicit contextual cuing effects based on the semantic category of the context words could be obtained. The semantic category membership of the context words either did or did not predict the location of the target. For instance, in a context composed of names of mammals, the target was always on the left side of the display, whereas in a context composed of fruitsvegetables, the target could appear randomly on the left or the right side. Experiments 3 and 4 tested the role of selective attention in semantic implicit learning and its expression. The experimental procedures were derived from Jiang and Chun's (2001) and Jiang and Leung's (2005) studies. Participants had to search for a target word written in green, for example, among green and red words. The green words all belonged to the same semantic category and constituted the attended context. The red words all belonged to some other semantic category and constituted the ignored context. During the search task, either the attended context or the ignored context predicted the target location. To separate the effect of selective attention on learning from its effect on the expression of the resulting knowledge, Experiment 4 included a transfer phase in which the colors of the contexts were switched-attended contexts became ignored and ignored contexts became attended.

\section{Experiment 1}

Experiment 1 investigated whether implicit contextual cuing effects could be based on the semantic category of a lexical context. The contextual cuing paradigm was set up with displays composed of words. The category membership of the context words did or did not predict the target location. In the visual search task, the participants were instructed to detect the name of a garment or building in a set of context words. In other words, the target was not a specific word but a word belonging to one of two categories. Categories were chosen instead of specific words because our pilot experiments had suggested that using particular target words (e.g., duck or hare) made it difficult to obtain contextual cuing effects based on the semantic properties of the words, perhaps because searching for a specific word triggers a "superficial" visual search, primarily based on its perceptual characteristics. The use of two categories to describe the target forced the participants to process the display items semantically to find the target. The target word was located in a limited area on the right or left side of the display (see Figure 1).

In each trial, the target word was presented among a set of context words all belonging to the same semantic category (i.e., mammals, trees-flowers, birds, or fruits-vegetables). The context words were distributed randomly throughout the display. The visual search task consisted of several blocks of trials, each block containing both predictive and nonpredictive trials. In predictive trials, the semantic category of the context words predicted the area where the target was located. For instance, when the context

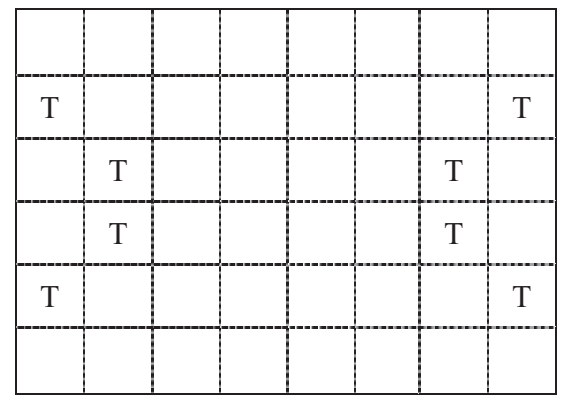

Figure 1. Target locations (T) in the invisible grid of Experiments 1 and 2.

was composed of names of mammals, the target was always located on the left side of the display; when it was composed of bird names, the target was always located on the right side of the display (see Figure 2).

In nonpredictive trials, the semantic category of the context did not predict the area where the target was located. For example, when the context was composed of names of fruits and vegetables, the target could appear on the left or right side of the display (likewise when the context was made up of names of trees and flowers). The visual search task thus involved two predictive semantic categories (predictive trials), systematically associated with a particular side of the target location, and two nonpredictive semantic categories (nonpredictive trials), in which the target could be equally located on the right or left side of the display. The search task was followed by a verbalization-then-recognition task aimed at testing the explicit versus implicit nature of the knowledge acquired during the search task.

\section{Method}

\section{Participants}

Twenty-four students at the University of Provence, Marseille, France (19 women and 5 men), whose mean age was 22.8 years $(S D=3$, range $=18-26$ years $)$, participated in the experiment. They all had normal or corrected-to-normal vision and felt motivated to participate in the experiment. None of the participants had any knowledge of the purpose of the study or the methods used.

\section{Apparatus}

The experiment was implemented in Psyscope software (Cohen, MacWhinney, Flatt, \& Provost, 1993) and run on a portable Power Book G4 computer (Apple Inc., Cupertino, CA) with a 15-inch $(38.1-\mathrm{cm})$ screen. The participants were seated approximately 50 $\mathrm{cm}$ from the screen. The stimuli were words written in uppercase letters (Courier New font, size 16) and displayed on the screen in an invisible eight-column six-row grid. This made 48 possible item locations. The grid subtended $28 \mathrm{~cm}$ horizontally and $18 \mathrm{~cm}$ vertically.

\section{Materials}

Targets. Each trial contained a single target that belonged to one of two possible semantic categories. The target could be either the name of a building or the name of a garment. There were 32 

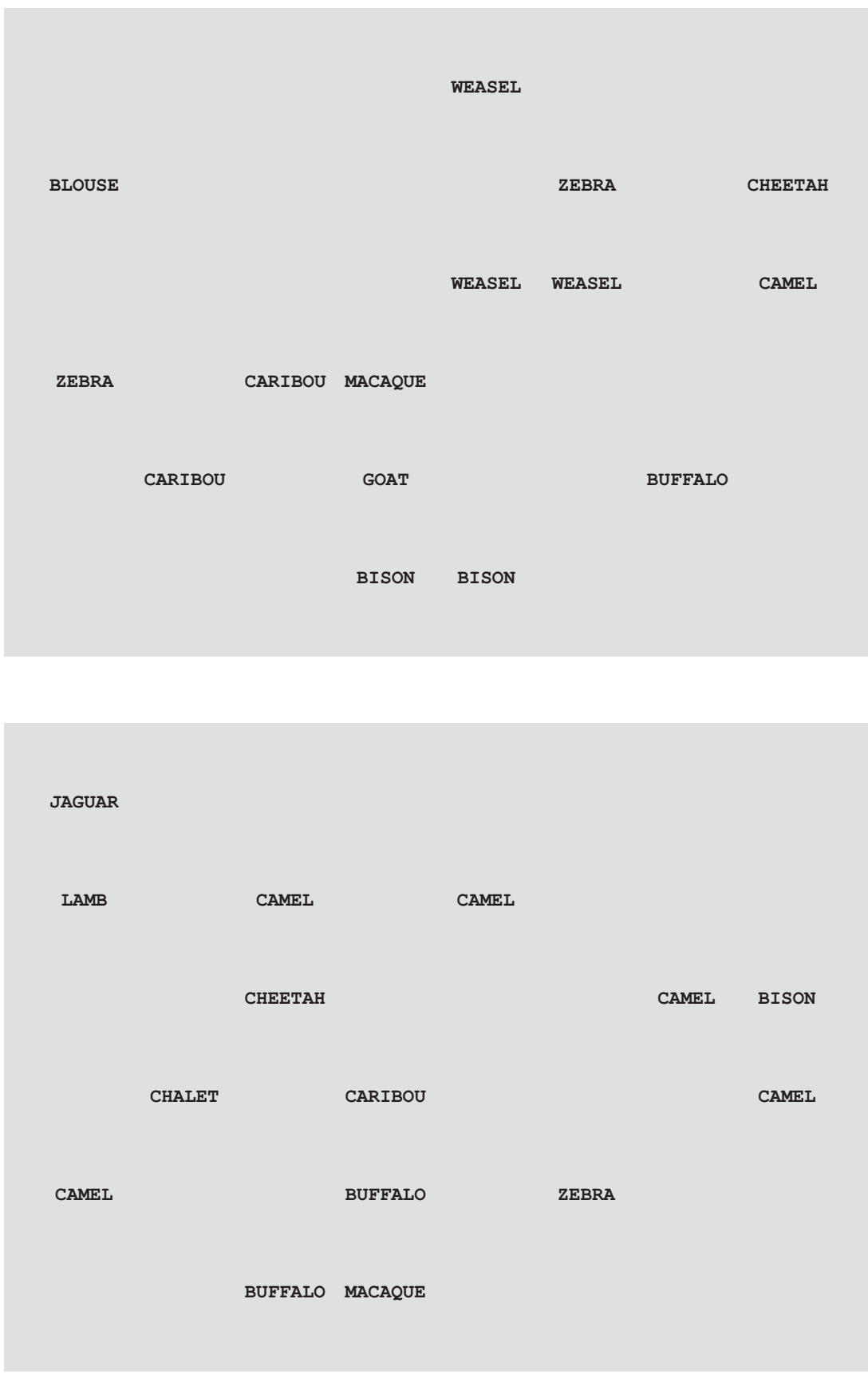

Figure 2. Example of two predictive trials for the association mammal-target on left used in Experiments 1 and 2. The target words BLOUSE and CHALET are surrounded by names of mammals. Words are translated from original French material.

possible targets, 16 for each semantic category (see Appendix A). All targets were familiar French words, and the category to which they belonged was considered unambiguous. The 32 targets were presented by circular permutation in such a way that they were displayed an equal number of times in each condition. On a given trial, the target could appear in one of the eight locations shown in Figure 1.

Contexts. Each search area contained 14 context words. The 14 words all belonged to the same semantic category, which contrasted sharply to the two categories chosen for the targets. There were four context categories-mammals, birds, treesflowers, and fruits-vegetables-with 13 words per category. The context words and their frequencies are listed by category in Appendix B (first set of lists). Analyses conducted on the printed and verbal frequencies (New, Pallier, Ferrand, \& Matos, 2001) of the words yielded no significant differences between these lists The length of the words was controlled in each list. For a given trial, the 14 context words were randomly drawn with replacement 
from one of the four category lists. So, a given context word could appear several times in a display. The 14 context words were distributed randomly across the invisible $8 \times 6$ grid. All words (14 context words plus the target word) were displayed in black on a gray background in Courier New font, size 16.

\section{Procedure}

The experiment lasted about 45 min and included two tasks: a visual search task and a verbalization-then-recognition task.

Search task. The participants were instructed to look as quickly as possible for the name of a garment or building in the display. As soon as they found the name, they had to press a designated key on the keyboard if it was a building and a different designated key if it was a garment. The appropriate response keys were counterbalanced across participants. The instructions also told the participants that every trial would always have either the name of a garment or the name of a building but never both.

The search task consisted of 24 blocks of 16 trials, making a total of 384 trials. Each block contained 8 predictive trials and 8 nonpredictive trials. In predictive trials, the category of the context predicted the area where the target was located (e.g., on the left side in a mammals context, on the right in a fruits-vegetables context). In nonpredictive trials, the category of the context did not predict the target location. The predictive and nonpredictive categories were counterbalanced across participants. Thus, each category was predictive for half of the participants and nonpredictive for the other half. The target location (left vs. right) for each predictive category was also counterbalanced across participants. Each predictive category could be associated with left locations (for a quarter of the participants) or with right locations (for a quarter of the participants). Potential target effects were neutralized by using a circular permutation design based on the two experimental factors of block and condition. For instance, a particular target presented in a predictive trial in a given block was presented in a nonpredictive trial in the next block, and vice versa. The order of circular permutations was varied between participants.

The experiment began with the instructions, which were followed by a practice block of eight nonpredictive trials aimed at familiarizing participants with the procedure. Immediately afterward, the participants performed the search task composed of 24 blocks of 16 trials. Within a block, the 16 trials were presented randomly. Participants pressed a button to start the first block. After a 500-ms delay, a stimulus pattern appeared on the screen. Participants had to search for the target (i.e., the name of a garment or building), and, as soon as the target was found, they had to quickly press the designated key for that target. The participant's response immediately triggered the 1-s display of a white screen with a black fixation point in the middle, after which the computer initiated the next trial. In the instructions, the participants were told to stare at this point after each trial. A break was programmed at the end of each block. Participants could go on to the next block or extend the break as they pleased.

Verbalization-then-recognition task. After the search task, the participants were asked the following three questions orally: "Did you notice any regularities in the material?" "Did you try to find any regularities and memorize certain contexts?" and "Did you notice any associations between the context and the target location during the experiment?" If the participants said they had not noticed any of the expected regularities, then they were given the recognition task. Participants had not been informed in advance about the recognition task. The recognition task consisted of a new block of 32 trials, 16 of which were generated in the same way as in the search task; that is, 8 were predictive and 8 were nonpredictive. There were also 8 counterpredictive trials and 8 nonpredictive filler trials. In the counterpredictive trials, the target was located on the opposite side from where it had been on the visual search task (e.g., if on the search task the target in predictive mammal contexts was located in the right part of the display, then the target was now located in the left part). The 8 nonpredictive filler trials were added simply to balance the number of predictive and nonpredictive contexts in the recognition task. The participants were instructed to look at the display on each of the 32 trials and answer the following question: "Do you have a feeling of déjà vu concerning the association between the target location and the context words? In other words, does the association between the target location and the context words look familiar with respect to the search phase?" No time limit was set for answering.

\section{Results}

\section{Search Task}

The error rate was below $1.61 \%$ for both predictive and nonpredictive trials, so the errors are not discussed. Our analyses thus pertain solely to RT on correct answers. RTs above or below the mean plus 3 standard deviations were discarded from the analyses. This procedure eliminated $1.41 \%$ of the correct answer RTs. The correct answer RTs were grouped into six epochs, each covering four consecutive blocks of trials. For each participant, a separate correct answer RT mean was calculated for each epoch and each condition tested.

A repeated-measures analysis of variance (ANOVA) was conducted, with condition (predictive vs. nonpredictive context) and epoch (1-6) as within-subject factors. The mean RTs for each condition and epoch are presented in Figure 3. The ANOVA yielded an epoch effect, $F(5,115)=37.17, M S E=.16, \eta^{2}=.62$, $p<.001$; no condition effect, $F(1,23)=1.89, M S E=.19, \eta^{2}=$ $.08, p=.183$; and an interaction effect between epoch and condition, $F(5,115)=2.81, M S E=.08, \eta^{2}=.11, p<.05$. Planned comparison at each epoch revealed a significant condition effect only on Epoch $3, F(1,23)=7.98, M S E=.12, \eta^{2}=.26, p<.01$. However, a significant contextual cuing effect was observed on Epoch 3 for predictive mammals contexts $(386 \mathrm{~ms}), F(1,11)=$ 5.92, MSE $=.15, \eta^{2}=.35, p<.05 ;$ predictive birds contexts $(411$ $\mathrm{ms}), F(1,11)=5.01, M S E=.20, \eta^{2}=.31, p<.05$; and predictive fruits-vegetables contexts $(241 \mathrm{~ms}), F(1,11)=5.24$, $M S E=.07, \eta^{2}=.32, p<.05$, but not for predictive trees-flowers contexts $(67 \mathrm{~ms}), F(1,11)<1, M S E=.35, \eta^{2}=.01$ (see Appendix $\mathrm{C}$ for the results in each category).

To quantify the trend toward faster learning in predictive than in nonpredictive contexts, we estimated the parameters of the power functions that fit the mean RT data in each condition. Like Chun and Jiang (2003), we used a power function because this yielded the best fits for our data set. The power function takes the form, $\mathrm{RT}=a+b N^{-c}$, where RT is the search response time, $N$ is the epoch number, constant $a$ represents the asymptote of learning, 


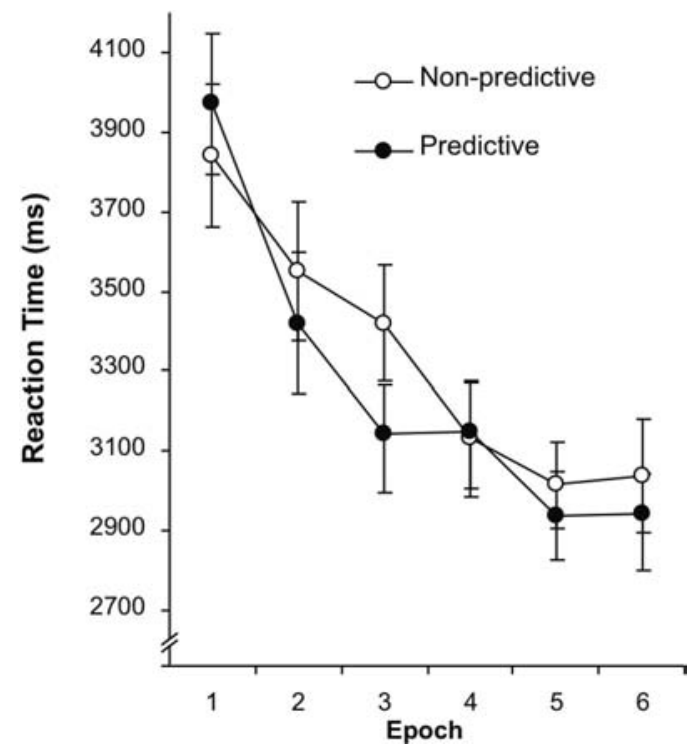

Figure 3. Mean target detection reaction time (in milliseconds) in the predictive and nonpredictive conditions of Experiment 1 by epoch. Error bars represent the standard error of the mean $(N=24)$.

constant $b$ is the difference between initial and asymptotic performance, and exponent $c$ is the learning rate. In fitting the data, we used the same constraints as Chun and Jiang: asymptotic performance $(a)$ had to be greater than 0 , the final level of asymptotic performance had to be equal in the predictive and nonpredictive conditions, and the learning parameter $(c)$ had to be less than or equal to 1 . The functions obtained from the mean group data were as follows: in the predictive condition, $\mathrm{RT}=2,535+1,437^{-0.716}$, with $r^{2}=.98$, and in the nonpredictive condition, $\mathrm{RT}=2,535+$ $1,354^{-0.532}$, with $r^{2}=.93$. So the learning rate was 0.716 in the predictive condition and 0.532 in the nonpredictive condition.

\section{Verbalization-Then-Recognition Task}

None of the participants reported having searched for regularities in the material or having tried to memorize the words, nor did any of them report having noticed the expected regularities, thus all participants performed the recognition task. A repeatedmeasures ANOVA on the percentage of déjà-vu responses, with condition as a within-subject variable, indicated no difference between the predictive (46\%), nonpredictive (54\%), and counterpredictive $(55 \%)$ conditions, $F(2,46)=1.62, M S E=366, \eta^{2}=$ $.07, p=.209$.

\section{Discussion}

These results show that a contextual cuing effect can be obtained with lexical material in a situation in which the semantic category of the context predicts the target location. A facilitating effect on the visual search was observed in the predictive condition as compared with the nonpredictive condition, but statistical analyses indicated that the effect was significant only on Epoch 3. This advantage was nevertheless significant for three of the four categories used in the experiment. Moreover, slopes estimated from the learning curves showed that the learning rate (parameter $c$ ) was larger in the predictive condition (0.716) than in the nonpredictive condition (0.532). Why did the apparently robust contextual cuing effect observed on Epoch 3 tend to disappear on the following epochs? This result (which we have already obtained in several unpublished experiments) could be due to a change of strategy during the search task. At the beginning of the task, participants do not know what the potential targets or context words will be. They are thus forced to process the items semantically to find the target. However, in the course of the search task, the same targets keep coming back in a cyclical way, and the context words are always drawn from the same lists. We can therefore hypothesize that participants may rapidly start storing information about the potential targets and contextual elements in working memory. This would allow them to categorize the items in "target versus context" terms and to do the search without having to access any semantic categories. Moreover, the potential target locations were limited to two areas of the display. It seems probable that, as the participants gradually detected this regularity, they processed fewer and fewer context words and this caused a concomitant decrease in contextual cuing (Chun \& Jiang, 1998, Experiment 4).

The verbalization-then-recognition task revealed the implicit nature of the learning that occurred during the search task - after the search, participants were not able to report any contextual regularities, nor were they able to use these regularities to differentiate predictive trials from nonpredictive or counterpredictive ones on the recognition task. However, this experiment does not allow us to firmly conclude that what took place here was implicit learning based on the semantic category of the context and not the mere learning of the particular contextual elements used. Indeed, the context words were drawn randomly (with replacement) from specific lists. The learning could thus have been based on the specificity of the context words, not on their semantic category membership. To find out whether the observed implicit learning can be undeniably ascribed to the categorical properties of the context, a transfer phase was implemented in Experiment 2. Throughout the search task, the same categories predicted the target location, but starting on the transfer phase, the context words were drawn from different word lists than those used on previous trials. Given that Experiment 1 yielded a significant contextual cuing effect on Epoch 3, the transfer phase was implemented at that point.

\section{Experiment 2}

Experiment 2 was designed to make sure that a contextual cuing effect could be obtained when only the semantic category of the context words predicted the target location. To test this, we set up a transfer phase starting on Epoch 3 of the search task. The method was the same as in Experiment 1 except that starting on Epoch 3, the context words were drawn from different lists than those used on Epochs 1 and 2. So, if a contextual cuing effect is obtained on Epoch 3, then it will be undeniably based on the semantic category of the context words and not on their specific features, notably their perceptual features.

\section{Method}

\section{Participants}

Fifty-six students at the University of Provence, Marseille, France (43 women and 13 men), whose mean age was 23.4 years 
( $S D=4$, range $=18-45$ years), participated in the experiment. They all had normal or corrected-to-normal vision and felt motivated to take part in the experiment. None of the participants had any knowledge of the purpose of the study or the methods used.

\section{Apparatus}

The experimental setup was the same as in the preceding experiment.

\section{Materials}

The targets were defined in the same way as in Experiment 1 . The context words belonged to the same four categories, but for each category, an additional list of 13 words was developed. These words and their frequencies are presented in Appendix B (second set of lists).

\section{Procedure}

Search task. The instructions and general principle of the task were the same as in Experiment 1. The experiment consisted of 16 blocks of 16 trials grouped into four epochs. During the first 8 blocks (Epochs 1 and 2), the context words were drawn randomly, with replacement, from one set of lists (e.g., the first set). During the last 8 blocks (Epochs 3 and 4), the context words were drawn randomly, with replacement, from the other set of lists (e.g., the second set). The list presentation order (first set then second set vs. second set then first set) was counterbalanced across participants within each experimental condition. The experimental procedure was the same as in Experiment 1.

Verbalization-then-recognition task. The task was the same as in Experiment 1.

\section{Results}

\section{Search Task}

In both the predictive and nonpredictive trials, the error rates were below $1.53 \%$. Correct RTs above and below the mean plus 3 standard deviations were discarded $(1.65 \%)$. A repeated-measures ANOVA was conducted, with condition (predictive vs. nonpredictive trial) and epoch (1-4) as within-subject factors. The RTs of the four epochs are plotted in Figure 4. The results yielded an epoch effect, $F(3,165)=80.45, M S E=.14, \eta^{2}=.59, p<.001$; no condition effect, $F(1,55)=2.92, M S E=.12, \eta^{2}=.05, p=$ .093; and an Epoch $\times$ Condition interaction, $F(3,165)=3.74$, $M S E=.10, \eta^{2}=.06, p<.05$. Planned comparisons revealed a significant condition effect on Epoch 3, $F(1,55)=9.05, M S E=$ $.11, \eta^{2}=.14, p<.01$, and on Epoch $4, F(1,55)=4.29, M S E=$ $.08, \eta^{2}=.08, p<.07$, suggesting contextual cuing during the transfer phase.

To make sure that the effect observed on Epoch 3 was not due to new learning but was the result of learning done during Epochs 1 and 2, we assessed the effect of the condition factor by analyzing only the first half of the Epoch 3 trials. This analysis showed that there was already a significant difference between the predictive $(3,314 \mathrm{~ms})$ and nonpredictive $(3,609 \mathrm{~ms})$ conditions in the first half of Epoch 3, $F(1,55)=5.99, M S E=.41, \eta^{2}=.10, p<.05$.

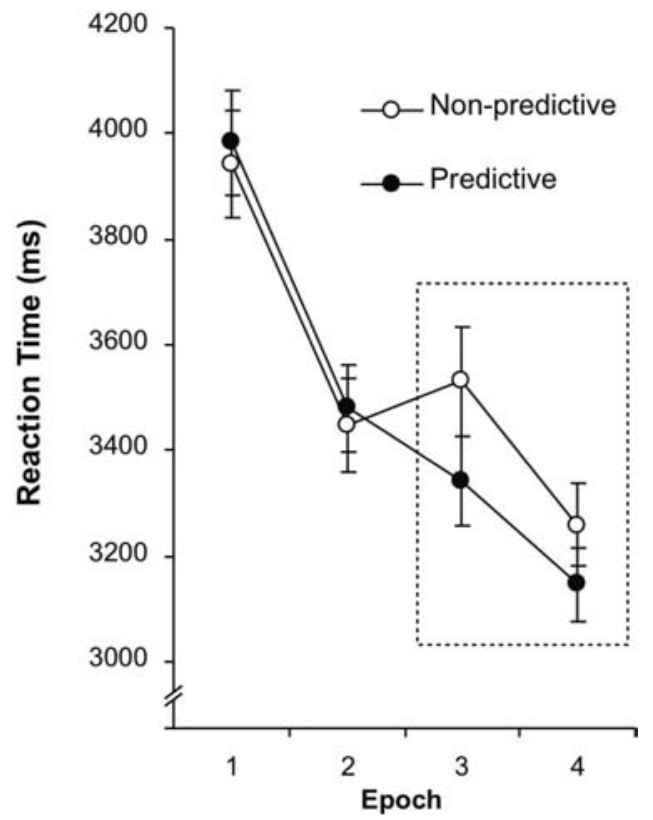

Figure 4. Mean target detection reaction time (in milliseconds) in the predictive and nonpredictive conditions of Experiment 2 by epoch. The error bars represent the standard error of the mean $(N=56)$. Epochs 3 and 4 correspond to the transfer phase.

\section{Verbalization-Then-Recognition Task}

None of the participants reported having looked for regularities or trying to memorize the material, and none reported having noticed any contextual regularities. Thus, all participants performed the recognition task. The déjà-vu response percentages were analyzed using a repeated-measures ANOVA. The ANOVA indicated no difference between the déjà-vu percentages obtained in the predictive $(55 \%)$, nonpredictive $(58 \%)$, and counterpredictive $(53 \%)$ conditions, $F(2,110)<1, M S E=444.7, \eta^{2}=.01$.

\section{Discussion}

This experiment was aimed at generalizing contextual cuing effects to cuing based on the semantic category of the elements that make up the target context. The results indicated a contextual cuing effect on Epoch 3, where the context words were not from the same lists as those used in Epochs 1 and 2. A detailed analysis of the results obtained for Epoch 3 showed that the facilitation observed during the transfer phase was significant, even during the first two blocks of trials. This finding suggests that learning did in fact occur during Epochs 1 and 2 because it showed up as soon as the transfer phase began. Undeniably, then, the learning noted on Epoch 3 was based on the semantic category of the context words and not on their specific features. Moreover, the data for each predictive category suggest that all of the four categories tend to produce better learning than the nonpredictive conditions (see Appendix $\mathrm{C}$ for the results in each predictive category).

Furthermore, at the end of the search task, none of the participants reported having noticed that certain contexts were associated with a particular target location. In addition, the recognition task results indicated no difference between the percentages of déjà-vu 
responses obtained in the predictive, nonpredictive, and counterpredictive conditions. This means that the learning effects observed on the search task were implicit in nature.

The results of Experiments 1 and 2 extend the implicit semantic contextual cuing effects obtained by Goujon et al. (2007). They confirm that implicit learning effects observed using the contextual cuing paradigm can be based on the semantic categorical properties of the context.

\section{Experiment 3}

The purpose of this experiment was to find out whether contextual cuing effects based on semantic category regularities in the target context require selective attention. The procedure of Experiment 1 was combined with the one used by Jiang and Chun (2001). The general principle was the same as in Experiment 1, except that here, the target (the name of a garment or building) was always displayed in green for half of the participants and always in red for the other half. On each trial, the target appeared among nine context words displayed in green and nine context words displayed in red. If the target was red, then the red context was the attended context and the green context was the ignored context; the reverse was true if the target was green. In a particular trial, the context words displayed in green all belonged to one semantic category and the red context words all belonged to another semantic category. The search task had three experimental conditions: attended predictive, ignored predictive, and nonpredictive. In attended predictive trials, the attended context (i.e., the semantic category of the context words written in the same color as the target) predicted the target location, but the ignored context (i.e., the semantic category of the context words written in a different color from the target) did not predict the target location. In ignored predictive trials, the attended context did not predict the target location, but the ignored context did. In nonpredictive trials, neither the attended context nor the ignored context predicted the target location. Figure 5 gives an example of two attended predictive trials and two ignored predictive trials.

To make the regularities more conspicuous, the target locations were limited to the far left and far right areas of the display. To minimize the potential use of a strategy consisting of confining the search to the right and left areas of the display, filler trials were added. In the filler trials, the target could be located anywhere in the display (see Figure 6).

\section{Method}

\section{Participants}

Thirty-six students or researchers at the University of Provence, Marseille, France (21 women and 15 men), whose mean age was 26 years $(S D=5$, range $=17-41$ years $)$, participated in the experiment. All had normal or corrected-to-normal vision. They all volunteered and felt motivated to participate in the experiment. None of them had any knowledge of the purpose of the study or the methods used.

\section{Apparatus}

The experimental setup was the same as in Experiment 1.

\section{Materials}

Targets. The targets were the same as those used in Experiment 1 (see Appendix A). However, this time, the target was written in green (RGB [red, green, blue]: $0,128,36$ ) for half of the participants and in red (RGB: 200, 50, 36) for the other half. The targets could appear in the eight possible locations shown in Figure 6.

Contexts. The target was displayed among 18 context words. Half of the context words were written in green and the other half were written in red. The green context words were drawn randomly, with replacement, from a list containing words from one category, and the red words were drawn randomly, with replacement, from a list of words from another category. Six semantic categories were used: mammals, birds, trees-flowers, fruitsvegetables, fish-seafood, and minerals-materials. The word lists used during this experiment and word frequencies are given in Appendix B. As a whole, words belonging to the mineralsmaterials category had a printed frequency significantly higher than the words belonging to the other categories and a verbal frequency significantly higher than the words belonging to the trees-flowers category (New et al., 2001). There were no significant differences between the other lists. Among these six categories, two were predictive: one attended predictive and one ignored predictive. The other four categories were nonpredictive: two attended nonpredictive and two ignored nonpredictive. An additional category, office supplies, was used for filler trials (see Appendix D).

\section{Procedure}

Search task. The participants were instructed to look as quickly and accurately as possible for the name of a garment or building, always written in green for half of the participants and always in red for the other half. The search task consisted of 24 blocks of 16 trials, making a total of 384 trials. Each block contained 4 attended predictive trials, 4 ignored predictive trials, 4 nonpredictive trials, and 4 filler trials. On attended predictive trials, words written in the attended color (e.g., green if the target was green) belonged to the attended predictive category: For example, if the attended context belonged to the predictive category mammals, then the target always appeared on the left side of the display. Words written in the ignored color (red) belonged to another category that did not predict the target's location (i.e., an ignored nonpredictive category). On ignored predictive trials, it was the opposite: Words written in the ignored color (e.g., red if the target was green) belonged to the ignored predictive category. For example, if the ignored context belonged to the predictive category birds, then the target always appeared on the right side of the display. Words written in the attended color (green) belonged to a category that did not predict the target's location (i.e., an attended nonpredictive category). On nonpredictive trials, words shown in the attended color belonged to an attended nonpredictive category, and words shown in the ignored color belonged to an ignored nonpredictive category. On these trials, the target could be located on the left or right side of the display. To make the regularities more conspicuous, the target locations were always on the far left or far right of the display.

All six categories appeared with the same frequency within a block. The categories attributed to each experimental condition were counterbalanced across participants, as were the target locations (left vs. right) for each attended or ignored predictive category. The target color, green or red, was counterbalanced across 


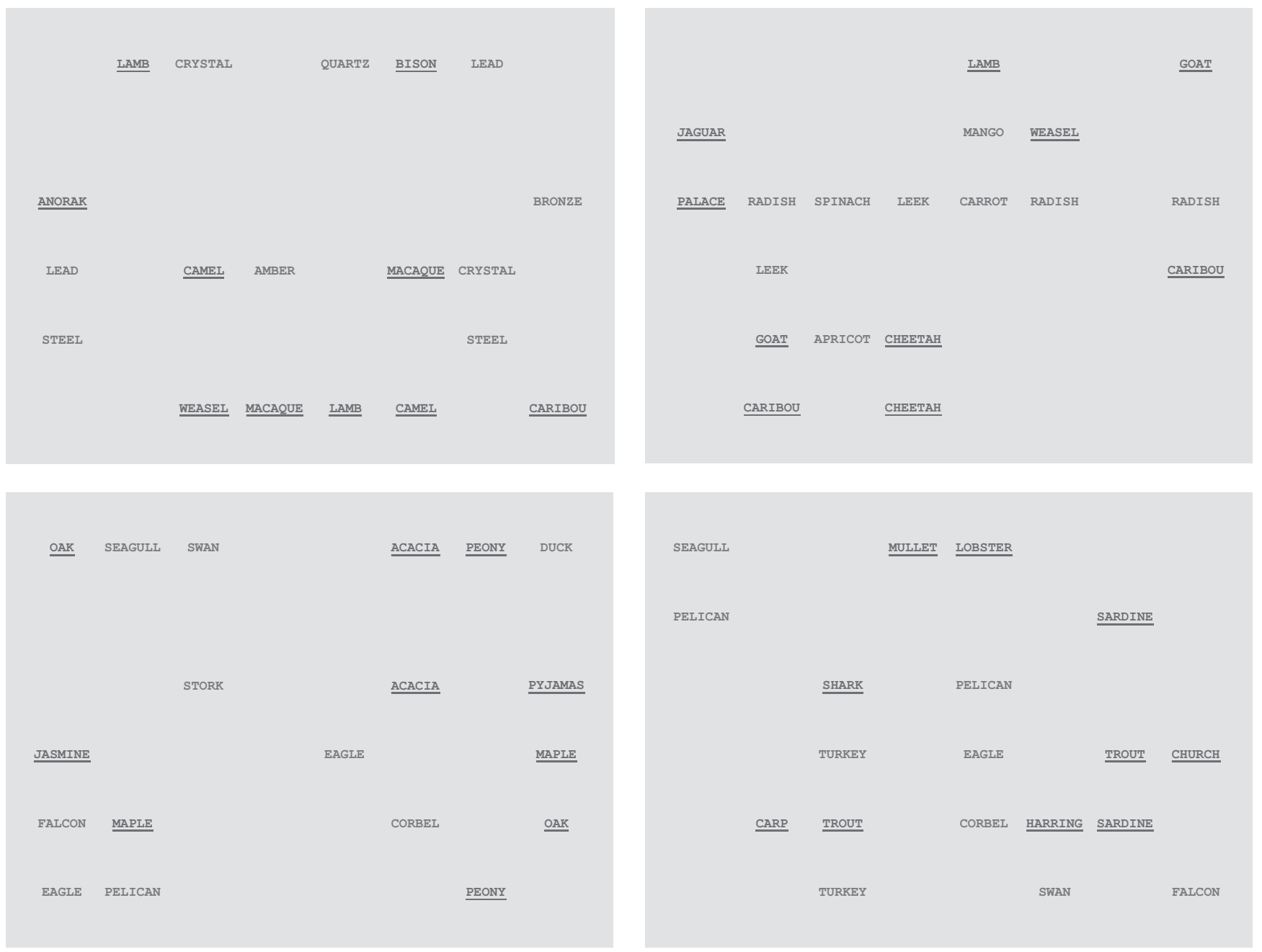

Figure 5. Top row: Example of two attended predictive trials for the mammals-target on left association. The target words ANORAK and PALACE were displayed in green among names of mammals in green (attended predictive category), and names of minerals-materials or fruits-vegetables were displayed in red (ignored nonpredictive categories). Bottom row: Example of two ignored predictive trials for the birds-target on right association. The target words PYJAMAS and $C H U R C H$ were displayed in green among names of birds in red (ignored predictive category) and names of trees-flowers or fish-seafood in green (attended nonpredictive categories). The words are translated into English from the original French material. Here, words displayed in green are underlined and words displayed in red are not.

participants. On filler trials, the target was displayed among names of office supplies and could be located anywhere in the display (see Figure 6).

The experimental procedure was the same as in Experiment 1 except for the instructions and the practice phase. The instructions informed participants that the search displays were composed of red and green words. If the target was green, then they were asked to search for the name of a garment or the name of a building among green words only. It was specified that the target was always written in green, never in red. Conversely, if the target was displayed in red, then they were instructed to search for the target among red words only. On the practice trials, the context words belonged to the filler category.

Verbalization-then-recognition task. The principle was the same as in the preceding experiments. However, this time, the

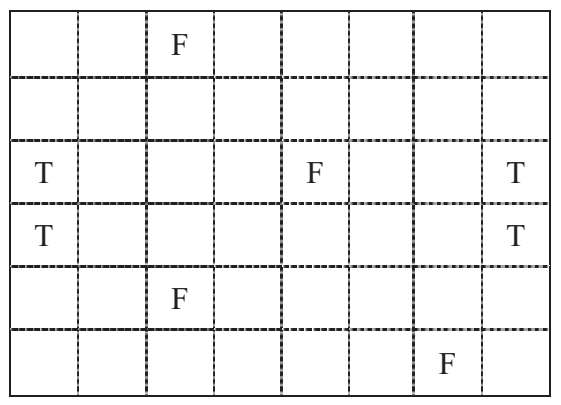

Figure 6. Target locations used in the invisible grid of Experiments 3 and 4. $\mathrm{T}=$ possible target locations on test trials; $\mathrm{F}=$ possible target locations on filler trials. 
recognition task consisted of 24 trials: 4 attended predictive, 4 attended counterpredictive, 4 ignored predictive, 4 ignored counterpredictive, and 8 nonpredictive. The attended and ignored counterpredictive trials were generated in the same way as the counterpredictive trials of Experiment 1. The instructions were the same as in Experiment 1.

\section{Results}

\section{Search Task}

The RTs obtained on filler trials were discarded. In the attended predictive, ignored predictive, and nonpredictive trials, the error rates were below $1.43 \%$. Correct RTs above and below the mean plus 3 standard deviations were discarded $(0.97 \%)$. The blocks of trials were grouped into six epochs, each epoch including four blocks. The RTs of the six epochs are plotted in Figure 7. A repeated-measures ANOVA was conducted, with condition (attended predictive, ignored predictive, and nonpredictive) and epoch (1-6) as within-subject factors. There was an epoch effect, $F(5,175)=69.82, M S E=.28, \eta^{2}=.67, p<.001 ;$ no condition effect, $F(2,70)=1.77, M S E=.47, \eta^{2}=.05, p=.18$; and no Epoch $\times$ Condition interaction, $F(10,350)=1.47, M S E=.13$, $\eta^{2}=.04, p=.15$. However, a repeated-measures ANOVA conducted with condition (attended predictive and nonpredictive) and epoch (1-6) revealed a condition effect, $F(1,35)=6.31, M S E=$ $.26, \eta^{2}=.15, p<.05$; an epoch effect, $F(5,175)=56.22, M S E=$ $.22, \eta^{2}=.62, p<.001$; and an Epoch $\times$ Condition interaction, $F(5,175)=2.33, M S E=.13, \eta^{2}=.06, p<.05$. Planned comparisons conducted with the attended predictive and nonpredictive condition on each epoch revealed a condition effect on Epoch 2, $F(1,35)=5.93, M S E=.16, \eta^{2}=.14, p<.05$; on Epoch 3, $F(1,35)=7.12, M S E=.14, \eta^{2}=.17, p<.05$; and on Epoch 5, $F(1,35)=8.10, M S E=.06, \eta^{2}=.19, p<.01$. Other comparisons (ignored predictive vs. nonpredictive and attended

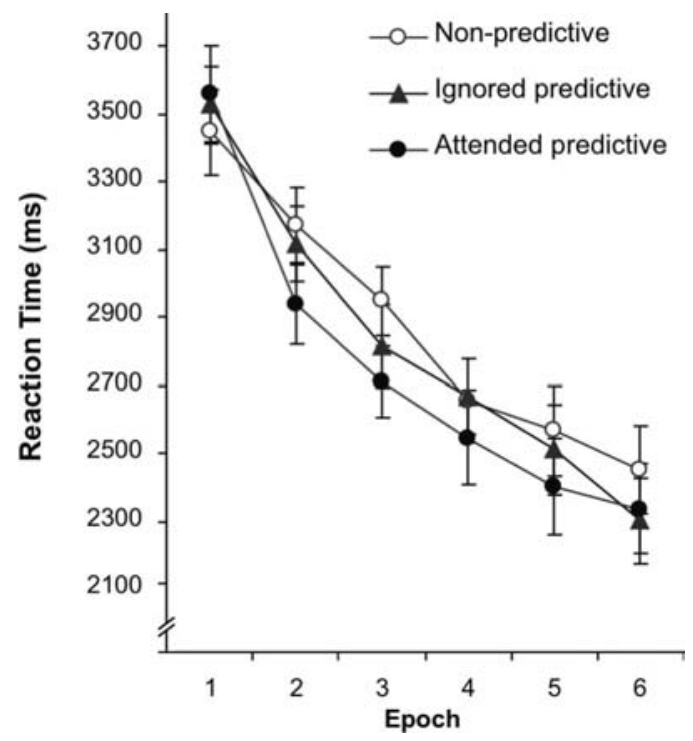

Figure 7. Mean target detection reaction time (in milliseconds) in the predictive and nonpredictive conditions of Experiment 3 by epoch. Error bars represent the standard error of the mean $(N=36)$. predictive vs. ignored predictive) did not indicate any condition effects (all $F \mathrm{~s}<1$ ) or Epoch $\times$ Condition interactions, $F(5$, $175)=1.22, M S E=.11, \eta^{2}=.03, p=.301$, and $F(5,175)<1$, $M S E=.15, \eta^{2}=.03$, respectively. The power functions fitting to the mean RT data for each condition were as follows: RT (attended predictive) $=1,429+2,126^{-0.475}, r^{2}=100$; RT (ignored predictive) $=1,429+2,154^{-0.428}, r^{2}=97$; and RT (nonpredictive) $=1,429+2,089^{-0.359}, r^{2}=95$.

\section{Verbalization-Then-Recognition Task}

None of the participants reported having noticed the expected regularities, trying to find regularities, or memorizing the material, thus all participants performed the recognition task. A repeatedmeasures ANOVA on the percentages of déjà-vu responses was conducted. It yielded no difference between the déjà-vu percentages in the attended predictive $(53 \%)$, attended counterpredictive (55\%), ignored predictive (60\%), ignored counterpredictive (52\%), and nonpredictive $(51 \%)$ conditions, $F(4,140)<1, M S E=$ 524.26, $\eta^{2}=.03$. However, pairwise comparisons of the different conditions showed that the ignored predictive and nonpredictive conditions were significantly different, $F(1,35)=4.90, M S E=$ $323.10, \eta^{2}=.12, p<.05$, and that the difference between the ignored predictive and ignored counterpredictive conditions was marginally significant, $F(1,35)=3.50, M S E=357.14, \eta^{2}=.09$, $p=.068$.

\section{Discussion}

The results indicated a contextual cuing effect when the semantic category of the attended context predicted the target location, even though the ignored context was nonpredictive. Furthermore, they did not indicate a significant difference between the ignored predictive and nonpredictive conditions. It is nevertheless worth noting that from the standpoint of performance, the ignored predictive condition fell between the attended predictive and nonpredictive conditions. The learning rates $(0.475$ for the attended predictive condition, 0.428 for the ignored predictive condition, and 0.359 for the nonpredictive condition) support this pattern. However, the statistical analyses do not allow us to conclude that there was a contextual cuing effect in the ignored predictive condition.

The results for the predictive attended condition confirm the cuing effect obtained in Experiment 1. However, compared with Experiment 1, the facilitation obtained in the attended predictive condition seems to be more robust. This difference could be due to certain changes in the experimental procedure. First, to make the regularity more conspicuous in the test trials, we limited the target locations to the far left and far right sides of the display. This may have optimally directed the participant's attention to the target location. However, of more importance, filler trials were added to make the potential locations of the target cover the entire display. The filler trials may also have minimized the use of a strategy that confined the search to the far left and far right sides of the display. Thus, a large number of contextual elements may have been processed in the course of the task, which could account for why this contextual cuing effect lasted until Epoch 6 (see Chun \& Jiang, 1998). Moreover, even if certain attended predictive categories were more conductive to a contextual cuing effect (e.g., mammals 
and birds), all of the six attended predictive categories used in this experiment seemed to bring about a benefit in RT with regard to the nonpredictive condition (see Appendix $\mathrm{C}$ for the results of each attended and ignored predictive category).

The results of the verbalization-then-recognition task once again pointed out the implicitness of the learning observed in the attended predictive condition. Not only did none of the participants report seeing the manipulated regularities, but also no difference was found on the recognition task between the attended predictive, nonpredictive, and attended counterpredictive conditions. Quite surprisingly, though, the ignored predictive trials were judged significantly more familiar than the nonpredictive trials and marginally more familiar than the ignored counterpredictive trials, even though no significant contextual cuing effect was observed. This result suggests that learning may nevertheless have taken place on the ignored predictive trials.

From these results, it seems that selective attention is necessary - or at least favorable to-contextual cuing based on the regularities manipulated in this experiment. Indeed, attended predictive contexts clearly triggered contextual cuing effects, whereas ignored predictive contexts did not, or not so strongly, with ignored predictive trials falling between attended predictive and nonpredictive trials. The results of the recognition task nevertheless suggest that learning may have occurred on the ignored predictive trials. Jiang and Leung's (2005) study helps account for these seemingly contradictory results. These authors showed (using a spatial contextual cuing task) that whereas expression of the implicit knowledge depends on our attending to the context, learning of the context itself is independent of attention. Indeed, their results did not provide evidence of contextual cuing effects in ignored predictive contexts. In contrast, with a transfer phase in which previously ignored predictive contexts suddenly became attended, these authors obtained immediately facilitated performance. They concluded that latent learning took place during ignored predictive trials. This kind of learning would not require allocating attention to contexts and would not show up directly in the form of contextual cuing effects. By contrast, when attended predictive contexts suddenly became ignored, the benefit immediately went away. This result suggests that attention is necessary to the expression of implicit knowledge. Experiment 4 was conducted in view of testing this phenomenon in a semantic implicit learning framework.

\section{Experiment 4}

The main goal of Experiment 4 was to test the impact of selective attention on the learning of semantic contextual regularities and the role of attention on the expression of this kind of learning. As in Jiang and Leung's (2005) study, a transfer was established. Contextual categories that were attended at the onset of the search task suddenly became ignored on the transfer phase. Conversely, the ignored categories suddenly became attended. Thus, the colors of the contextual categories were switched. It is important to note that the color of the target did not change during the transfer phase. Given that Epoch 3 turned out to be the one most conducive to obtaining a contextual cuing effect, the transfer phase was implemented on Epoch 3. For example, if the mammals category was an attended context (e.g., green if the target was green) on Epochs 1 and 2, then it became an ignored context (e.g., red) on Epochs 3 and 4. Throughout the search task, the same categories predicted the target location (e.g., mammals-target on left and birds-target on right), but the colors in which they were displayed were switched at the beginning of Epoch 3 .

If selective attention is required for regularities to be learned, then no contextual cuing effect should be observed for predictive contexts that suddenly become attended on Epoch 3, after two epochs during which these contexts were ignored (i.e., ignoredthen-attended predictive condition). If selective attention is required for learning to show up, then no contextual cuing effect should be observed for the predictive contexts that suddenly become ignored on Epoch 3, after two epochs during which they were attended (i.e., attended-then-ignored condition).

In parallel, we also wanted to make sure that the tested effects were undeniably based on the context's semantic category regularities. Thus, following the same principle as in Experiment 2, the words used on Epochs 3 and 4 were different from those used on Epochs 1 and 2.

\section{Method}

\section{Participants}

Thirty-six students or researchers at the University of Provence, Marseille, France (19 women and 17 men), whose mean age was 26 years $(S D=6$, range $=17-42$ years $)$, participated in the experiment. All had normal or corrected-to-normal vision. None of the participants had any knowledge of the purpose of the study or the methods used.

\section{Apparatus}

The experimental setup was the same as in Experiment 1.

\section{Materials}

The materials and categories were the same as in Experiment 3 except that each context was composed of 22 words (11 red words and 11 green words) and that two lists of 13 words were generated for each of seven categories. The lists are presented in Appendixes $\mathrm{B}$ and $\mathrm{D}$.

\section{Procedure}

Search task. The instructions and the general principle were the same as in Experiment 3, but the task consisted of 16 blocks of 16 trials and included a transfer phase starting on Block 9. The transfer pertained to both the context words (see Experiment 2) and their colors. The task was divided into four epochs, each containing 4 blocks of trials. As in Experiment 2, during the first 8 blocks (Epochs 1 and 2), the context words were drawn from one set of lists (e.g., the first set). During the last 8 blocks (Epochs 3 and 4), the context words were drawn from the other set of lists (e.g., the second set). The list presentation order was counterbalanced across participants. In parallel, the contexts' colors were switched at the beginning of Epoch 3, such that ignored contexts became attended and attended contexts became ignored. So, the category defining the attended predictive contexts on Epochs 1 and 2 defined the ignored predictive contexts on Epochs 3 and 4, and vice versa (category defining the ignored predictive contexts became the attended predictive condition). The two attended nonpre- 
dictive categories became the ignored nonpredictive categories, and the two ignored nonpredictive categories became the two attended nonpredictive categories. This made three experimental conditions: attended-then-ignored predictive, ignored-thenattended predictive, and nonpredictive. As in the preceding experiment, the contexts attributed to each experimental condition were counterbalanced across participants. The experimental procedure was the same as in Experiment 3.

Verbalization-then-recognition task. The task was the same as in Experiment 3 except that contexts displayed on Epochs 1 and 2 and contexts displayed on Epochs 3 and 4 were used. The recognition task thus consisted of 48 trials.

\section{Results}

\section{Search Task}

The responses on the filler trials were discarded. The error rates were below $1.46 \%$, so as above, the error data were not analyzed. RTs on errors, and all RTs above or below the mean plus 3 standard deviations, were discarded from the analyses. This procedure eliminated about $1.28 \%$ of the correct answer RTs. The RTs of the four epochs are plotted in Figure 8. A repeatedmeasures ANOVA was conducted, with condition (attended-thenignored predictive, ignored-then-attended predictive, and nonpredictive) and epoch (1-4) as within-subject factors. There was an epoch effect, $F(3,105)=32.97, M S E=.44, \eta^{2}=.49, p<.001$; no condition effect, $F(2,70)<1, M S E=.87, \eta^{2}=.02$; and no Epoch $\times$ Condition interaction, $F(6,210)=1.17, M S E=.21$, $\eta^{2}=.03, p=.326$.

However, planned contrasts revealed a significant difference between the ignored-then-attended predictive and the nonpredictive conditions on Epoch 3, $F(1,35)=5.63, M S E=.22, \eta^{2}=.04$,

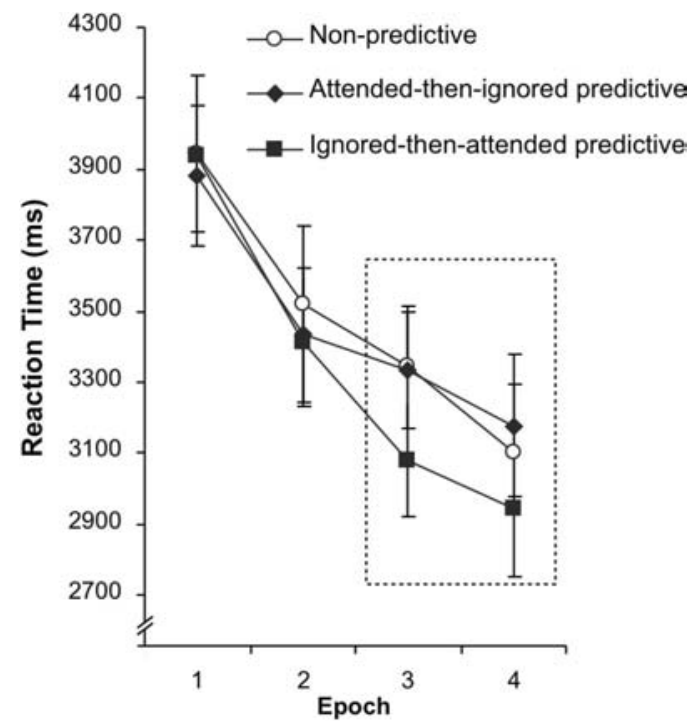

Figure 8. Mean target detection reaction time (in milliseconds) in the attended-then-ignored predictive, ignored-then-attended predictive, and nonpredictive conditions of Experiment 4 by epoch. Error bars represent the standard error of the mean $(N=36)$. Epochs 3 and 4 correspond to the transfer phase. $p<.05$, whereas no difference was found between the attendedthen-ignored and the nonpredictive conditions, $F(1,35)<1$, $M S E=.20, \eta^{2}=.00$. As in Experiment 2, to make sure that the effect observed on Epoch 3 was not due to new learning but was instead the result of learning taking place during Epochs 1 and 2, the effect of the condition factor was assessed by analyzing only the first half of the Epoch 3 trials. The results showed that right from the beginning of Epoch 3, the ignored predictive condition that had become attended $(3,217 \mathrm{~ms})$ differed significantly from the nonpredictive condition $(3,606 \mathrm{~ms}), F(1,35)=4.34, M S E=$ $.63, \eta^{2}=.11, p<.05$. There was no difference between the attended-then-ignored predictive $(3,572 \mathrm{~ms})$ and nonpredictive $(3,606 \mathrm{~ms})$ conditions, $F(1,35)<1, M S E=.54, \eta^{2}=.00$.

\section{Verbalization-Then-Recognition Task}

None of the participants reported having noticed the expected regularities, and none reported having tried to find regularities or memorize the material. Thus, all participants performed the recognition task. The percentages of déjà-vu responses were analyzed in two repeated-measures ANOVAs. The first ANOVA compared the responses for the contexts used during Epochs 1 and 2 of the search task. The results indicated no difference between the déjà-vu percentages obtained in the attended predictive (44\%), attended counterpredictive (44\%), ignored predictive (44\%), ignored counterpredictive $(46 \%)$, and nonpredictive $(45 \%)$ conditions used before the transfer, $F(4$, $140)<1, M S E=638.4, \eta^{2}=.00$. The second ANOVA compared the responses obtained for the contexts used during Epochs 3 and 4. They indicated no overall difference between the attended predictive $(57 \%)$, attended counterpredictive $(60 \%)$, ignored predictive $(61 \%)$, ignored counterpredictive (49\%), and nonpredictive (53\%) conditions after the transfer, $F(4,140)=1.61, M S E=516.4, \eta^{2}=.04, p=.174$. However, specific contrasts showed that the ignored predictive condition differed significantly from the ignored counterpredictive condition, $F(1,35)=7.60, M S E=330.1, \eta^{2}=.18, p<.01$, and differed marginally from the nonpredictive condition, $F(1$, $35)=3.56, M S E=291.4, \eta^{2}=.09, p=.068$. There were no differences between the other experimental conditions.

\section{Discussion}

The main results of this experiment concern the transfer phase. They revealed a contextual cuing effect in the previously ignored predictive condition when it suddenly became attended but no contextual cuing effect in the previously attended condition when it suddenly became ignored. A detailed analysis of the results obtained for Epoch 3 showed that the overall facilitation observed in the newly attended condition was significant right from the very first trials of the transfer phase. This shows that the learning observed at the beginning of the transfer phase took place during previous epochs, that is, Epochs 1 and 2. This finding means that regularities were learned before the transfer, when the predictive contexts were being ignored by participants. A benefit over nonpredictive condition was observed on Epoch 3 for all suddenly attended predictive categories except fish-seafood $(-45 \mathrm{~ms} ; 574 \mathrm{~ms}$ for mammals, $338 \mathrm{~ms}$ for trees-flowers, $294 \mathrm{~ms}$ for birds, $218 \mathrm{~ms}$ for fruits-vegetables, 
and $199 \mathrm{~ms}$ for minerals-materials). It is important to note that if word frequency seems to partially influence the initial performance level, then it does not seem to influence the contextual cuing effects. For instance, the minerals-materials predictive category was not more conducive to the emergence of a contextual cuing effect than were the other predictive categories, even during the transfer phase where the contextual words were new for the participants.

By contrast, the previously attended condition did not exhibit a contextual cuing effect when it suddenly became ignored. This means that learning was not manifested on ignored contexts. The use of new words during the transfer phase proves that these effects result from the semantic properties of the contexts. This experiment thus suggests that latent learning took place in contexts ignored during Epochs 1 and 2. However, for this learning to be expressed, attention had to be focused on the contextual regularities.

The results of the recognition task showed that attended predictive trials on the second part of the search task were not judged more familiar than were attended nonpredictive or counterpredictive trials. However, in line with the results of Experiment 3 , the ignored predictive trials used in the second part of the search were judged significantly more familiar than the ignored counterpredictive trials and marginally more familiar than the nonpredictive trials. This unexpected result suggests that search task performance and recognition task performance are not necessarily correlated.

Thus, it seems that implicit learning of semantic regularities does not require attention to be focused on contextual regularities. In contrast, the expression of implicit knowledge appears to be dependent on selective attention being directed at the regularities. These results corroborate those obtained by Jiang and Leung (2005) using spatial layouts and thereby extend this aspect of implicit learning to semantic regularities.

\section{General Discussion}

During the exploration of a visual environment, the cognitive system has the ability to implicitly learn exhibited regularities (e.g., Chun \& Jiang, 1998). The present study offers some new findings on the implicit learning mechanisms involved in visual search tasks. The main contributions of the experiments conducted here can be summarized in three points. First, contextual cuing effects can be based on semantic category regularities in the context. Second, these effects can result from implicit learning. Last, although selective attention specifically directed at semantic regularities seems to be necessary for a semantic contextual cuing effect to show up, latent learning can nevertheless take place even if attention is not focused on the regularities.

\section{Contextual Cuing Based on Semantic Regularities}

The four experiments conducted in this study provided evidence of contextual cuing effects based on the semantic properties of contexts composed of words. In Experiments 1 and 3, visual search was facilitated when the semantic category of the context predicted the target location. However, because the context words were always drawn from the same lists, these results can be interpreted equally well in terms of learning based on the semantic categorical properties of the context and in terms of specific feature learning. To make up for this drawback, Experiments 2 and 4 included a transfer phase during which the words were drawn from lists other than the ones used at the beginning of the experiment. A contextual cuing effect was obtained right from the very first trials of the transfer, indicating that the learning was based on semantic categorization.

However, it is difficult to completely exclude the hypothesis that the guidance of attention lies on nonsemantic features that covary with the semantic categories that were manipulated. In the past, it has been shown that some results from implicit learning tasks, first interpreted as semantic effects, could also be explained by the participants' sensitivity to certain regularities that covaried with the rule being manipulated (e.g., Bright \& Burton, 1994; Churchill \& Gilmore, 1998; Newell \& Bright, 2002). Even if observed contextual cuing effects cannot be interpreted in terms of frequency or length of the items forming the different categories, it is not excluded that other nonsemantic properties could differentiate these categories. Nevertheless, when we broke the results down by category, benefit effects were observed at a descriptive level for nearly all the categories used in each experiment (inferential statistics were rarely significant because of the small number of participants in each condition, e.g., 6 participants for Experiment 3 and Experiment 4 for each predictive category). Even if certain categories seem to be more conducive to the emergence of a contextual cuing effect than others, contextual cuing effects are not due to only one category but seem to emerge from each category used in that study. As a whole, it would seem that regardless of the frequency of the words, the most homogeneous categories (here, mammals and birds) are the ones most likely to trigger a semantic contextual cuing effect. It can be hypothesized that one of the determining factors of semantic contextual cuing is the strength of the semantic links among category members. However, to our knowledge, there are no French databases containing psycholinguistic indexes that could be used to validate this hypothesis. Other hypotheses, related, for instance, to the familiarity of the categories (regardless of the familiarity of the items composing each category) or their age of acquisition, still remain.

\section{Semantic Contextual Cuing and Spatial Contextual Cuing}

To what extent can contextual cuing based on semantic properties be compared with contextual cuing based on spatial repetitions? Given substantial differences between the methods used in classical experiments and in the experiments of the present study, it is difficult to strictly compare the strength of our effects with those observed in the literature, both in terms of learning speed (measured by $c$ ) and in terms of magnitude (advantage of the predictive condition over the nonpredictive condition). First of all, although the magnitude of the mean maximal observed benefit was greater in our study (about $200 \mathrm{~ms}$ ) than in classical experiments (about $100 \mathrm{~ms}$ ), the initial performance level was also much higher. Thus, these values have to be weighted, taking into account the initial performances. In Experiments 1 and 3, the improvement 
was about $5 \%-6 \%{ }^{1}$ higher in the predictive condition than in the nonpredictive condition, compared with about $7 \%$ in spatial cuing tasks (e.g., Chun \& Jiang, 1998, Experiment 1). The observed benefit therefore appears relatively comparable. Second, whereas our learning rates were closer to those reported by Chun and Jiang (2003), the number of exposures required on predictive trials to obtain contextual cuing effects based on semantic aspects (about 20 on average) was far higher that those generally observed (usually about five repetitions of an old configuration). Moreover, the emergence of these effects seems to vary across categories. For example, the mammals category seems to trigger earlier contextual cuing effect than the trees-flowers category. Thus, although semantic contextual cuing effects involving categorization and generalization seem to emerge later than spatial contextual cuing effects based on specific properties, we cannot defend this point of view with certainty because of the large differences in the methods used.

\section{Semantic Contextual Cuing Results From Implicit Learning}

A crucial aspect of the semantic contextual cuing effects observed in our study is that they were the outcome of implicit learning. Again, implicit learning refers to an adaptation process through which the behavior of an individual becomes sensitive to a structure in an incidental manner and without the individual being able to verbally report or even consciously access the resulting knowledge (Reber, 1989). In the experiments conducted in the present study, both the acquisition process and the resulting knowledge were implicit. Not only did none of the participants say, after the search task, that they had looked for regularities or tried to memorize the experimental material, but also no one was able to verbalize the contextual regularities that had facilitated their search, even though the experimenter asked them to report those regularities. Moreover, on the recognition task, the predictive trials that had been subject to a contextual cuing effect were not found to be more familiar by participants than the nonpredictive or counterpredictive trials. These results indicate that the learning manifested via contextual cuing effects on the various search tasks in our study was implicit in nature - in addition to the fact that the participants did not learn the regularities deliberately, they were not even aware that they had knowledge of them.

These findings extend those obtained previously using numerical displays with contextual regularities based on the evennessoddness of the numbers used (Goujon et al., 2007). The present study with lexical materials generalizes these results to other types of semantic categories. The results show that contextual cuing effects based on semantic regularities are not necessarily associated with awareness of the regularities. Awareness that comes with the learning of regularities in real-world scenes (Brockmole \& Henderson, 2006a, 2006b) does not pertain solely to the presence of semantic cues. This study thus provides additional empirical arguments in support of the idea of implicit conceptual learning. Implicit learning, then, is not confined to taking specific features into account - notably perceptual features of the context - but can also be based on more conceptual properties, namely, the semantic category membership of the contextual elements (for a review, see Reber, 1989).
It is important to note, however, that in our study, participants were not learning new abstract knowledge, as argued in the literature on implicit learning (cf. Reber, 1989), but rather were learning to associate some conceptual knowledge already in memory (e.g., the concept of mammal) with a target position (for a discussion of this type of effect, see Didierjean, 2007). Thus, the cognitive system would be able to learn new knowledge from preexisting semantic knowledge and extend it to new exemplars belonging to the same semantic category. This type of effect is probably quite different from one involving complex rules that participants might learn implicitly through passive exposure to the material. A question that arises is whether that associative learning really involves a process of abstraction. According to us, the results reported here do not prove the existence of an unconscious system allowing elaboration of new conceptual and abstract knowledge but are in favor of a cognitive unconscious, operating on semantic knowledge preexisting in long-term memory.

\section{Contextual Cuing and Selective Attention}

The third main result of our study concerns the role of selective attention in semantic contextual cuing. Experiments 3 and 4 showed that selective visual attending to contextual elements was necessary for the expression of implicit learning but that latent learning could still take place in a passive way. In Experiment 3, selective attending to contextual regularities was needed to obtain a contextual cuing effect. When the predictive category appeared in an ignored color (i.e., in a color different from the target), there was no significant benefit on RT, whereas a contextual cuing effect was obtained when the participants were attending to the color of the predictive category (i.e., the target color). In Experiment 4, to distinguish between the role of selective attention in learning and in its expression, we included a transfer phase in the search task (see Jiang \& Leung, 2005). Previously attended categories became ignored on the transfer, and previously ignored categories became attended. The data showed that previously attended predictive contexts did not trigger a contextual cuing effect when they suddenly became ignored. This result suggests that selective attention is required for learning to be expressed. However, previously ignored predictive contexts did trigger an immediate cuing effect when they suddenly became attended. The participants thus seem to have learned the regularities in ignored contexts, but this learning could not show up unless selective attention was directed at the contextual elements. The use of new words on the transfer phase proves that these effects were rooted in the categorical and semantic regularities of the context.

The present study suggests that implicit learning of semantic regularities can occur even though attention is not directly focused on those regularities. However, for the resulting knowledge to show up on a contextual cuing task, it seems that visual attention must be directed at the predictive regularities. Although these results are consistent with those obtained by Jiang and Leung (2005) on spatial contexts, they are nonetheless unexpected, given the depth of processing required to detect semantic category regularities.

\footnotetext{
${ }^{1}$ Improvement was measured in the following way: [RT minimum (nonpredictive condition) - RT minimum (predictive condition)] / RT initial. Used values were approximate.
} 
Although it is often claimed that learning cannot take place on objects unless attention is focused on them (for a review, see Chun $\&$ Turk-Browne, 2007), our results are in line with recent evidence showing that perceptual learning can occur in the absence of focused attention (Seitz \& Watanabe, 2003; Watanabe, Nanez, \& Sasaki, 2001). In those studies, series of experiments were conducted in which participants were repeatedly exposed to a weak background motion signal that was below their perceptual thresholds. This background motion was irrelevant to the central task in which the participant's attention was engaged. Participants became sensitive to the repetitive exposure to the motion signal, even when it was below the visibility threshold and irrelevant to the central task. These findings indicate that focused attention is not necessary for perceptual learning. Seitz and Watanabe (2003) found that the learning of task-irrelevant features depends on the temporal pairing between the presentation of the task-irrelevant stimulus and the task-relevant features: Task-irrelevant features have to coincide with the target presentation. It is important to note that in Experiments 3 and 4 of our study, attended and ignored contexts were always presented simultaneously. Seitz and Watanabe (2005) proposed a unified model for perceptual learning that attempts to explain both learning of task-target features and learning of taskirrelevant features. Namely, when signals that are triggered and diffused as a result of a task performance (reinforcement signals) and signals that are produced by presentation of task-relevant and task-irrelevant features (stimulus signals) temporally coincide, a long-term sensitivity enhancement to the stimuli occurs. The unified model is in line with Posner and Petersen's (1990) attentional model, which includes three subsystems: an orienting subsystem and an executive subsystem operating on task-relevant features and an alerting subsystem controlling a nonspecific arousal state. The alerting subsystem would enhance processing of a large extent of the scene, including task-irrelevant features. In agreement with this model, the categorical learning observed in our study on ignored words does not mean that no form of attention was engaged in the learning process but instead suggests that latent learning can nevertheless occur on conceptual regularities present outside the area of attentional focus. Our results open up the possibility of extending the unified model of Seitz and Watanabe (2005), aimed at accounting for perceptual learning, to accounting for categorical and semantic learning.

\section{Conclusion}

In the research on visual-scene perception, current theories (Irwin, 1991; Rensink, 2000; see, however, Hollingworth, 2004) defend the idea that our visual representations are local and transient. However, even if representations are sparse and start fading at the very instant they arise, the visual processing that takes place has an impact on memory and on the cognitive and visuomotor operations that ensue. The present study showed how attention directing is facilitated by implicit knowledge about semantic regularities in the environment. It also showed that this knowledge, inaccessible to awareness, can be acquired without selectively attending to the semantic aspects of elements present in the scene. The visuocognitive system thus seems to be capable of passively encoding not only perceptual information (Jiang \& Leung, 2005; for reviews, see Seitz \& Dinse, 2007; Seitz \& Watanabe, 2005; see, however, Chun \& Turk-Browne, 2007) but also semantic information in the environment. For learning to manifest during visual searching, it nevertheless seems that attention must be focused on semantic regularities. Implicit knowledge of semantic regularities in the surroundings may enter into the construction of scene schemas and thereby contribute to accounting for the adaptability of perceptual processing, in spite of the sparsity of our conscious representations.

\section{References}

Abrams, R. L., \& Greenwald, A. G. (2000). Parts outweigh the whole (word) in unconscious analysis of meaning. Psychological Science, 11, $118-124$.

Biederman, I., Mezzanotte, R. J., \& Rabinowitz, J. C. (1982). Scene perception: Detecting and judging objects undergoing relational violations. Cognitive Psychology, 14, 143-177.

Bright, J. E. H., \& Burton, A. M. (1994). Past midnight: Semantic processing in an implicit learning task. Quarterly Journal of Experimental Psychology: Human Experimental Psychology, 47(A), 71-89.

Brockmole, J. R., Castelhano, M. S., \& Henderson, J. M. (2006). Contextual cuing in naturalistic scenes: Global and local contexts. Journal of Experimental Psychology: Learning, Memory, and Cognition, 32, 699706.

Brockmole, J. R., \& Henderson, J. M. (2006a). Recognition and attention guidance during contextual cueing in real-world scenes: Evidence from eye movements. Quarterly Journal of Experimental Psychology, 59, $1177-1187$.

Brockmole, J. R., \& Henderson, J. M. (2006b). Using real-world scenes as contextual cues for search. Visual Cognition, 13, 99-108.

Chun, M. M., \& Jiang, Y. (1998). Contextual cueing: Implicit learning and memory of visual context guides spatial attention. Cognitive Psychology, $36,28-71$.

Chun, M. M., \& Jiang, Y. (1999). Top-down attentional guidance based on implicit learning of visual covariation. Psychological Science, 10, 360365.

Chun, M. M., \& Jiang, Y. (2003). Implicit, long-term spatial contextual memory. Journal of Experimental Psychology: Learning, Memory, and Cognition, 29, 224-234.

Chun, M. M., \& Turk-Browne, N. B. (2007). Interactions between attention and memory. Current Opinion in Neurobiology, 17, 177-184.

Churchill, E. F., \& Gilmore, D. J. (1998). Selection through rejection: Reconsidering the invariant learning paradigm. Quarterly Journal of Experimental Psychology: Human Experimental Psychology, 51(A), $1-17$.

Cleeremans, A., Destrebecqz, A., \& Boyer, M. (1998). Implicit learning: News from the front. Trends in Cognitive Sciences, 2, 406-416.

Cohen, J. D., MacWhinney, B., Flatt, M., \& Provost, J. (1993). PsyScope: A new graphic interactive environment for designing psychology experiments. Behavioral Research Methods, Instruments, and Computers, 25, 257-271.

Damian, M. F. (2001). Congruity effects evoked by subliminally presented primes: Automaticity rather than semantic processing. Journal of Experimental Psychology: Human Perception and Performance, 27, 154 165.

Didierjean, A. (2007). Parity effects in an implicit learning task. British Journal of Psychology, 98, 529-545.

Endo, N., \& Takeda, Y. (2004). Selective learning of spatial configuration and object identity in visual search. Perception \& Psychophysics, 66, 293-302.

Frensch, P. A., Lin, J., \& Buchner, A. (1998). Learning versus behavioral expression of the learned: The effects of a secondary tone-counting task on implicit learning in the serial reaction task. Psychological Research, 61, 83-98.

Frensch, P. A., Wenke, D., \& Rünger, D. (1999). A secondary tone- 
counting task suppresses expression of knowledge in serial reaction task. Journal of Experimental Psychology: Learning, Memory, and Cognition, 25, 260-274.

Friedman, A. (1979). Framing pictures: The role of knowledge in automatized encoding and memory for gist. Journal of Experimental Psychology: General, 108, 316-355.

Goujon, A., Didierjean, A., \& Marmèche, E. (2007). Contextual cueing based on specific and categorical properties of the environment. Visual Cognition, 15, 257-275.

Hayes, N. A., \& Broadbent, D. (1988). Two modes of learning for interactive tasks. Cognition, 28, 249-276.

Henderson, J. M., Weeks, P. A., Jr., \& Hollingworth, A. (1999). The effects of semantic consistency on eye movements during complex scene viewing. Journal of Experimental Psychology: Human Perception and Performance, 25, 210-228.

Hollingworth, A. (2004). Constructing visual representations of natural scenes: The roles of short- and long-term visual memory. Journal of Experimental Psychology: Human Perception and Performance, 30, 519-537.

Hollingworth, A., \& Henderson, J. M. (2002). Accurate visual memory for previously attended objects in natural scenes. Journal of Experimental Psychology: Human Perception and Performance, 28, 113-136.

Irwin, D. E. (1991). Information integration across saccadic eye movements. Cognitive Psychology, 23, 420-456.

Irwin, D. E., \& Andrews, R. V. (1996). Integration and accumulation of information across saccadic eye movements. In T. Unui \& J. L. Mcclelland (Ed.), Attention and Performance XVI: Information integration in perception and communication (pp. 125-155). Cambridge, MA: MIT Press.

Jiang, Y., \& Chun, M. M. (2001). Selective attention modulates implicit learning. Quarterly Journal of Experimental Psychology: Human Experimental Psychology, 54(A), 1105-1124.

Jiang, Y., \& Leung, A. W. (2005). Implicit learning of ignored visual context. Psychonomic Bulletin \& Review, 12, 100-106.

Jiang, Y., Song, J.-H., \& Rigas, A. (2005). High-capacity spatial contextual memory. Psychonomic Bulletin \& Review, 12, 524-529.

Jiang, Y., \& Wagner, L. C. (2004). What is learned in spatial contextual cuing-Configuration or individual locations? Perception \& Psychophysics, 66, 454-463.

Klauer, K. C., Eder, A. B., Greenwald, A. G., \& Abrams, R. L. (2007). Priming of semantic classifications by novel subliminal prime words. Consciousness and Cognition: An International Journal, 16, 63-83.

Knowlton, B. J., \& Squire, L. R. (1996). Artificial grammar learning depends on implicit acquisition of both abstract and exemplar-specific information. Journal of Experimental Psychology: Learning, Memory, and Cognition, 22, 169-181.

Mandler, J. M., \& Ritchey, G. H. (1977). Long-term memory for pictures. Journal of Experimental Psychology: Human Learning \& Memory, 3, 386-396.

Marcel, A. J. (1983). Conscious and unconscious perception: Experiments on visual masking and word recognition. Cognitive Psychology, 15, 197-237.

Naccache, L., \& Dehaene, S. (2001). Unconscious semantic priming extends to novel unseen stimuli. Cognition, 80, 223-237.

New, B., Pallier, C., Ferrand, L., \& Matos, R. (2001). Une base de données lexicale du français contemporain sur Internet: Lexique [A lexical da- tabase on the Internet about contemporary French: Lexique]. L'Année Psychologique, 101, 447-462.

Newell, B. R., \& Bright, J. E. H. (2002). Well past midnight: Calling time on implicit invariant learning. European Journal of Cognitive Psychology, 14, 185-205.

Olson, I. R., \& Chun, M. M. (2001). Temporal contextual cuing of visual attention. Journal of Experimental Psychology: Learning, Memory, and Cognition, 27, 1299-1313.

Ono, F., Jiang, Y., \& Kawahara, J.-i. (2005). Intertrial temporal contextual cuing: Association across successive visual search trials guides spatial attention. Journal of Experimental Psychology: Human Perception and Performance, 31, 703-712.

Perruchet, P., \& Pacteau, C. (1990). Synthetic grammar learning: Implicit rule abstraction or explicit fragmentary knowledge? Journal of Experimental Psychology: General, 119, 264-275.

Perruchet, P., \& Pacton, S. (2006). Implicit learning and statistical learning: One phenomenon, two approaches. Trends in Cognitive Sciences, 10, 233-238.

Perruchet, P., Vinter, A., Pacteau, C., \& Gallego, J. (2002). The formation of structurally relevant units in artificial grammar learning. Quarterly Journal of Experimental Psychology: Human Experimental Psychology, 55(A), 485-503.

Peterson, M. S., \& Kramer, A. F. (2001). Attentional guidance of the eyes by contextual information and abrupt onsets. Perception \& Psychophysics, 63, 1239-1249.

Posner, M. I., \& Petersen, S. E. (1990). The attention system of the human brain. Annual Review of Neuroscience, 13, 25-42.

Pothos, E. M. (2007). Theories of artificial grammar learning. Psychological Bulletin, 133, 227-244.

Potter, M. C. (1975, March 14). Meaning in visual search. Science, 187, 965-966.

Reber, A. S. (1989). Implicit learning and tacit knowledge. Journal of Experimental Psychology: General, 118, 219-235.

Remillard, G. (2003). Pure perceptual-based sequence learning. Journal of Experimental Psychology: Learning, Memory, and Cognition, 29, 581597.

Rensink, R. A. (2000). The dynamic representation of scenes. Visual Cognition, 7, 17-42.

Seger, C. A. (1994). Implicit learning. Psychological Bulletin, 115, $163-$ 196.

Seitz, A. R., \& Dinse, H. R. (2007). A common framework for perceptual learning. Current Opinion in Neurobiology, 17, 148-153.

Seitz, A. R., \& Watanabe, T. (2003, March 6). Is subliminal learning really passive? Nature, 422, 36.

Seitz, A. R., \& Watanabe, T. (2005). A unified model for perceptual learning. Trends in Cognitive Sciences, 9, 329-334.

Simons, D. J., \& Rensink, R. A. (2005). Change blindness: Past, present, and future. Trends in Cognitive Sciences, 9, 16-20.

Turk-Browne, N. B., Jungé, J., \& Scholl, B. J. (2005). The automaticity of visual statistical learning. Journal of Experimental Psychology: General, 134, 552-564.

Vokey, J. R., \& Brooks, L. R. (1994). Fragmentary knowledge and the processing-specific control of structural sensitivity. Journal of Experimental Psychology: Learning, Memory, and Cognition, 20, 1504-1510.

Watanabe, T., Nanez, J. E., \& Sasaki, Y. (2001, October 25). Perceptual learning without perception. Nature, 413, 844-848. 
Appendix A

Targets Used in Experiments 1-4

\begin{tabular}{ll}
\hline Garments & Buildings \\
\hline GILET & USINE \\
BOTTE & FERME \\
TRICOT & MAIRIE \\
VESTON & PALAIS \\
ANORAK & MOULIN \\
BLOUSE & CHALET \\
BONNET & GARAGE \\
MOUFLE & MANOIR \\
CORSET & ÉGLISE \\
PYJAMA & CABANE \\
MAILLOT & GYMNASE \\
CHEMISE & COLLÉGE \\
CULOTTE & AUBERGE \\
FOULARD & TAVERNE \\
COSTUME & MAGASIN \\
BERMUDA & CHÂTEAU \\
\hline
\end{tabular}

Appendix B

Lists of Context Words Used in Experiments 1-4

\begin{tabular}{|c|c|c|c|c|c|c|c|c|c|c|c|}
\hline \multicolumn{12}{|c|}{ First set of context word lists used in Experiments 1-4 } \\
\hline \multicolumn{3}{|c|}{ Fruits-vegetables $^{\mathrm{a}}$} & \multicolumn{3}{|c|}{ Trees-flowers ${ }^{\mathrm{b}}$} & \multicolumn{3}{|c|}{ Mammals $^{\mathrm{c}}$} & \multicolumn{3}{|c|}{ Birds $^{\mathrm{d}}$} \\
\hline Word & Ffilms & Fbook & Word & Ffilms & Fbook & Word & Ffilms & Fbook & Word & Ffilms & Fbook \\
\hline MELON & 4.22 & 5.27 & CHÊNE & 2.89 & 16.49 & BISON & 2.83 & 1.28 & AIGLE & 4.28 & 7.91 \\
\hline RADIS & 1.45 & 3.11 & FRÊNE & 0.00 & 1.62 & ZÈBRE & 1.33 & 3.04 & HÉRON & 0.18 & 1.08 \\
\hline COING & 0.06 & 0.41 & SAPIN & 4.10 & 9.86 & RENNE & 0.84 & 0.47 & CYGNE & 2.47 & 4.66 \\
\hline RAISIN & 4.76 & 4.86 & ACACIA & 0.06 & 3.24 & AGNEAU & 8.73 & 5.95 & CANARD & 17.11 & 16.15 \\
\hline ANANAS & 3.37 & 3.51 & CACTUS & 2.65 & 2.30 & CHÈVRE & 6.45 & 10.14 & PIVERT & 0.48 & 0.81 \\
\hline MANGUE & 0.30 & 0.74 & ÉRABLE & 1.99 & 1.15 & BUFFLE & 3.13 & 1.96 & FAISAN & 1.14 & 1.69 \\
\hline PAPAYE & 0.42 & 0.14 & JASMIN & 1.20 & 4.19 & JAGUAR & 1.02 & 0.41 & FAUCON & 9.04 & 2.36 \\
\hline FRAISE & 5.30 & 3.99 & MUGUET & 0.12 & 3.85 & COBAYE & 2.89 & 0.74 & DINDON & 1.27 & 0.74 \\
\hline POIREAU & 0.60 & 0.88 & FOUGËRE & 0.66 & 0.74 & BELETTE & 1.33 & 0.68 & CIGOGNE & 1.02 & 1.35 \\
\hline POTIRON & 0.96 & 0.61 & PIVOINE & 0.24 & 0.74 & CHAMEAU & 3.31 & 5.41 & PÉLICAN & 0.30 & 0.81 \\
\hline ÉPINARD & 0.12 & 0.41 & ROMARIN & 1.02 & 1.01 & GUÉPARD & 0.42 & 0.34 & CORBEAU & 3.86 & 4.19 \\
\hline CAROTTE & 4.10 & 2.97 & TILLEUL & 0.30 & 5.00 & MACAQUE & 1.57 & 0.14 & GOÉLAND & 0.30 & 0.81 \\
\hline ABRICOT & 0.36 & 1.15 & CAMÉLIA & 0.18 & 0.27 & CARIBOU & 0.48 & 0.14 & MÉSANGE & 0.06 & 6.89 \\
\hline \multicolumn{12}{|c|}{ Second set of context word lists used in Experiments 2 and 4} \\
\hline \multicolumn{3}{|c|}{ Fruits-vegetables $^{\mathrm{a}}$} & \multicolumn{3}{|c|}{ Trees-flowers $^{\mathrm{b}}$} & \multicolumn{3}{|c|}{ Mammals $^{\mathrm{c}}$} & \multicolumn{3}{|c|}{$\operatorname{Birds}^{\mathrm{d}}$} \\
\hline Word & Ffilms & Fbook & Word & Ffilms & Fbook & Word & Ffilms & Fbook & Word & Ffilms & Fbook \\
\hline FIGUE & 1.87 & 1.35 & CÈDRE & 0.54 & 2.16 & PANDA & 1.69 & 0.14 & MERLE & 1.39 & 2.64 \\
\hline PRUNE & 1.51 & 1.55 & HÊTRE & 0.18 & 3.38 & FURET & 0.66 & 0.14 & POULE & 26.63 & 16.82 \\
\hline NAVET & 1.39 & 0.88 & SAULE & 0.96 & 1.96 & TIGRE & 10.3 & 4.86 & HIBOU & 2.65 & 2.36 \\
\hline CASSIS & 0.30 & 3.45 & BAMBOU & 3.80 & 3.78 & BREBIS & 5.66 & 7.09 & CAILLE & 1.81 & 2.97 \\
\hline GOYAVE & 0.06 & 0.2 & CYPRÈS & 0.54 & 8.51 & BÉLIER & 1.87 & 3.11 & PIGEON & 9.58 & 7.97 \\
\hline CERISE & 2.77 & 3.31 & MIMOSA & 0.36 & 1.35 & COYOTE & 1.33 & 0.34 & PINSON & 0.54 & 1.08 \\
\hline BANANE & 8.98 & 4.19 & ROSEAU & 0.54 & 2.97 & GIRAFE & 2.11 & 1.89 & TOUCAN & 0.12 & 0.14 \\
\hline CITRON & 8.01 & 9.05 & TULIPE & 1.63 & 0.54 & CASTOR & 1.87 & 1.08 & RAPACE & 0.66 & 1.49 \\
\hline PRUNEAU & 0.96 & 1.35 & PALMIER & 1.45 & 3.04 & GAZELLE & 1.02 & 2.77 & FLAMANT & 0.12 & 0.07 \\
\hline AIRELLE & 0.12 & 0.07 & LAURIER & 0.36 & 3.58 & HAMSTER & 2.17 & 0.68 & PERDRIX & 1.14 & 1.49 \\
\hline HARICOT & 1.20 & 1.22 & LAVANDE & 1.02 & 6.82 & TAUREAU & 9.88 & 10.07 & MOINEAU & 1.93 & 4.32 \\
\hline POIVRON & 0.30 & 0.27 & PLATANE & 0.12 & 4.26 & BABOUIN & 1.39 & 0.54 & MOUETTE & 1.20 & 5.47 \\
\hline ASPERGE & 0.48 & 5.88 & SÉQUOIA & 0.18 & 1.22 & GORILLE & 7.29 & 2.03 & COLOMBE & 3.49 & 3.51 \\
\hline
\end{tabular}


Appendix B (continued)

\begin{tabular}{|c|c|c|c|c|c|}
\hline \multicolumn{6}{|c|}{ First set of context word lists used in Experiments 3 and 4} \\
\hline \multicolumn{3}{|c|}{ Fish-seafood $^{\mathrm{e}}$} & \multicolumn{3}{|c|}{ Minerals-materials ${ }^{\mathrm{f}}$} \\
\hline Word & Ffilms & Fbook & Word & Ffilms & Fbook \\
\hline BULOT & 0.06 & 0.00 & ACIER & 19.10 & 33.38 \\
\hline MORUE & 1.99 & 4.86 & PLOMB & 10.12 & 20.34 \\
\hline CARPE & 2.59 & 2.77 & AMBRE & 1.51 & 4.39 \\
\hline HOMARD & 5.12 & 3.51 & BRONZE & 6.93 & 18.58 \\
\hline REQUIN & 10.3 & 1.62 & TITANE & 2.05 & 0.20 \\
\hline HARENG & 1.69 & 2.91 & SAPHIR & 1.08 & 1.22 \\
\hline TRUITE & 1.39 & 3.38 & MARBRE & 4.82 & 41.49 \\
\hline ROUGET & 0.30 & 0.54 & QUARTZ & 0.42 & 1.82 \\
\hline SARDINE & 0.54 & 1.28 & BASALTE & 1.99 & 0.47 \\
\hline DAURADE & 0.18 & 0.74 & URANIUM & 0.06 & 0.95 \\
\hline LIMANDE & 0.00 & 0.61 & CRISTAL & 13.67 & 13.72 \\
\hline SEICHES & 0.24 & 0.68 & GOUDRON & 2.05 & 7.50 \\
\hline POULPES & 1.20 & 1.22 & PÉTROLE & 11.27 & 14.73 \\
\hline
\end{tabular}

Second set of context word lists used in Experiment 4

\begin{tabular}{|c|c|c|c|c|c|}
\hline \multicolumn{3}{|c|}{ Fish-seafood ${ }^{\mathrm{e}}$} & \multicolumn{3}{|c|}{ Minerals-materials ${ }^{\mathrm{f}}$} \\
\hline Word & Ffilms & Fbook & Word & Ffilms & Fbook \\
\hline MOULE & 4.04 & 3.99 & MÉTAL & 14.58 & 41.82 \\
\hline CRABE & 5.60 & 7.30 & RUBIS & 2.71 & 3.11 \\
\hline COLIN & 0.00 & 0.54 & BÉTON & 6.08 & 15.20 \\
\hline CALMAR & 1.20 & 0.27 & CUIVRE & 3.61 & 30.68 \\
\hline SAUMON & 5.18 & 3.65 & LAITON & 0.12 & 1.55 \\
\hline OURSIN & 0.06 & 0.54 & NICKEL & 1.63 & 1.22 \\
\hline MERLAN & 0.24 & 3.45 & ARGILE & 4.34 & 9.32 \\
\hline GARDON & 0.3 & 0.41 & TOPAZE & 0.18 & 0.81 \\
\hline ANCHOIS & 1.57 & 2.57 & GRANITE & 0.24 & 0.47 \\
\hline ESPADON & 1.08 & 0.47 & BAUXITE & 0.12 & 0.41 \\
\hline BROCHET & 0.24 & 3.18 & DIAMANT & 14.34 & 14.12 \\
\hline PIRANHA & 0.72 & 0 & MERCURE & 0.96 & 1.76 \\
\hline PIEUVRE & 1.99 & 1.82 & CHARBON & 6.51 & 22.03 \\
\hline
\end{tabular}

Note. Verbal frequencies (Ffilms) and printed frequencies (Fbook) were extracted from New, Pallier, Ferrand, and Matos (2001). The length of the words was controlled in each list.

${ }^{\mathrm{a}}$ For the first set of context word lists, mean Ffilms $=2.00$ and mean Fbook $=2.16$; for the second set of context word lists, Ffilms $=2.15$ and Fbook $=2.52 .{ }^{\mathrm{b}}$ For the first set of context word lists, mean Ffilms $=1.19$ and mean Fbook $=3.88$; for the second set of context word lists, Ffilms $=0.89$ and Fbook $=3.35 . \quad{ }^{\mathrm{c}}$ For the first set of context word lists, mean Ffilms $=2.64$ and mean Fbook $=2.36$; for the second set of context word lists, Ffilms $=3.63$ and Fbook $=2.67 .{ }^{\mathrm{d}}$ For the first set of context word lists, mean Ffilms $=3.19$ and mean Fbook $=3.80$; for the second set of context word lists, Ffilms $=3.94$ and Fbook $=3.87$. ${ }^{\mathrm{e}}$ For the first set of context word lists, mean Ffilms $=1.97$ and mean Fbook $=1.85$; for the second set of context word lists, Ffilms $=1.71$ and Fbook $=2.17$. ${ }^{\mathrm{f}}$ For the first set of context word lists, mean Ffilms $=5.77$ and mean Fbook $=12.21$; for the second set of context word lists, Ffilms $=4.26$ and Fbook $=11.00$. 


\section{Appendix C}

Results Relative to Each Predictive Category in Experiments 1-4
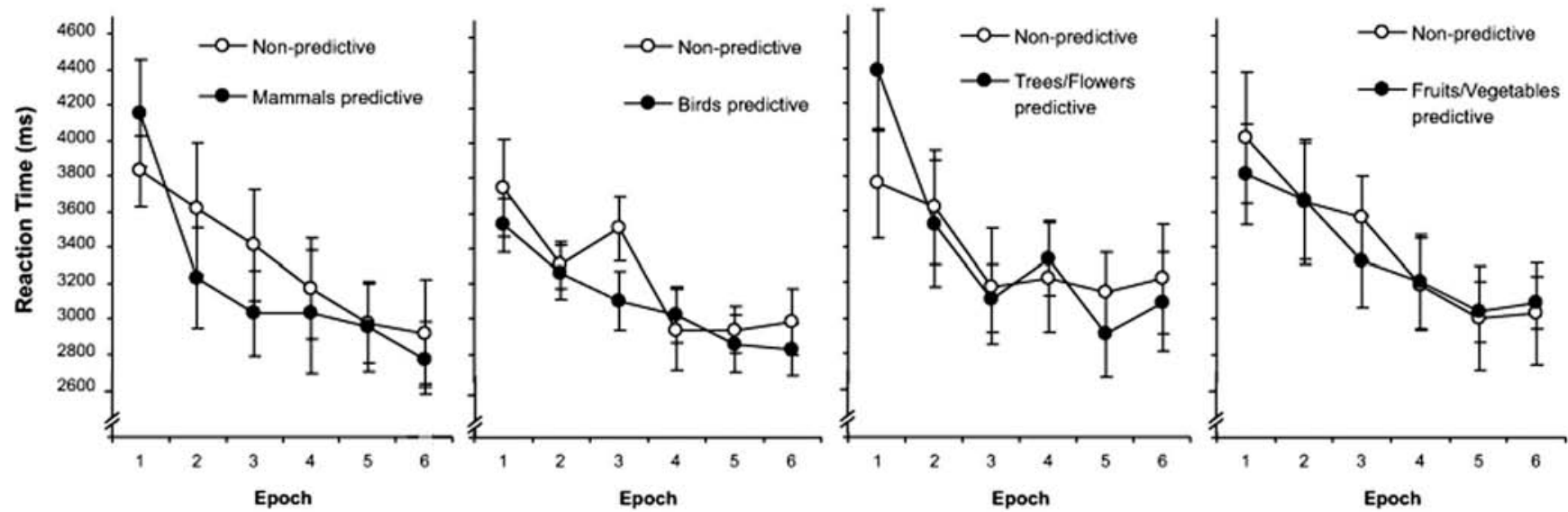

Figure C1. Results of Experiment 1. Mean target detection reaction time (in milliseconds) in the mammals predictive, birds predictive, trees-flowers predictive, and fruits-vegetables predictive conditions and in their matched nonpredictive conditions. The error bars represent the standard error of the mean $(N=12)$.
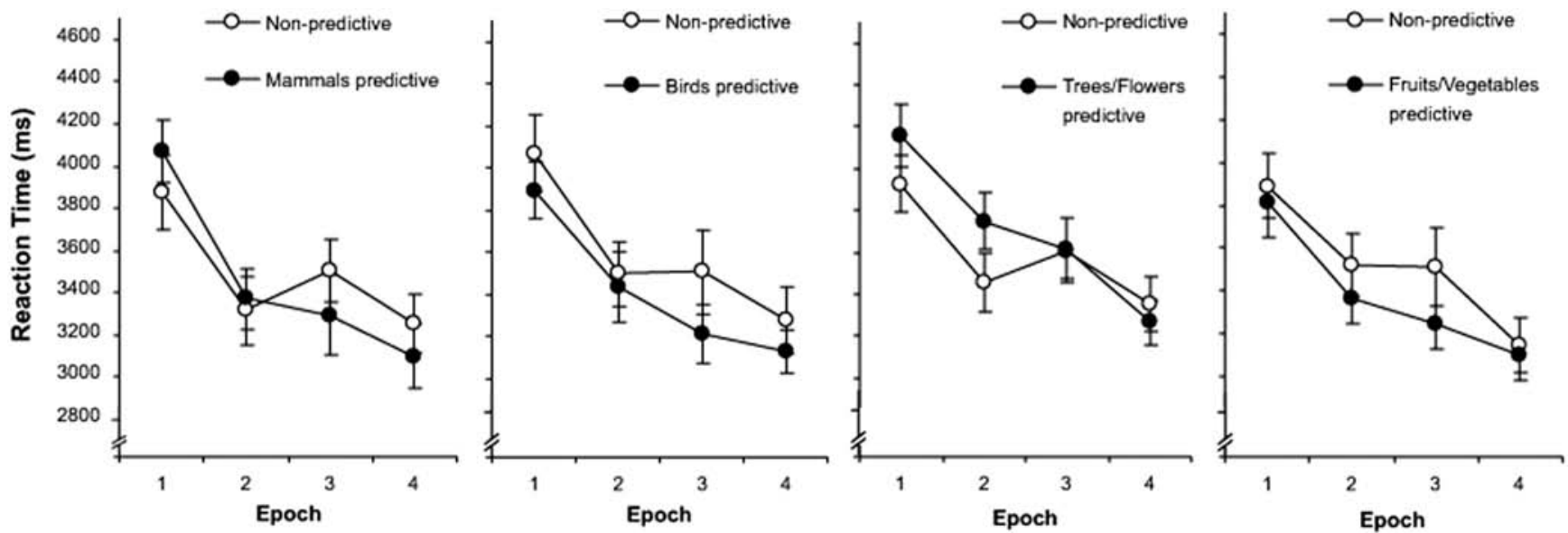

Figure C2. Results of Experiment 2. Mean target detection reaction time (in milliseconds) in the mammals predictive, birds predictive, trees-flowers predictive, and fruits-vegetables predictive conditions and in their matched nonpredictive conditions. The error bars represent the standard error of the mean $(N=28)$. 
Appendix C (continued)
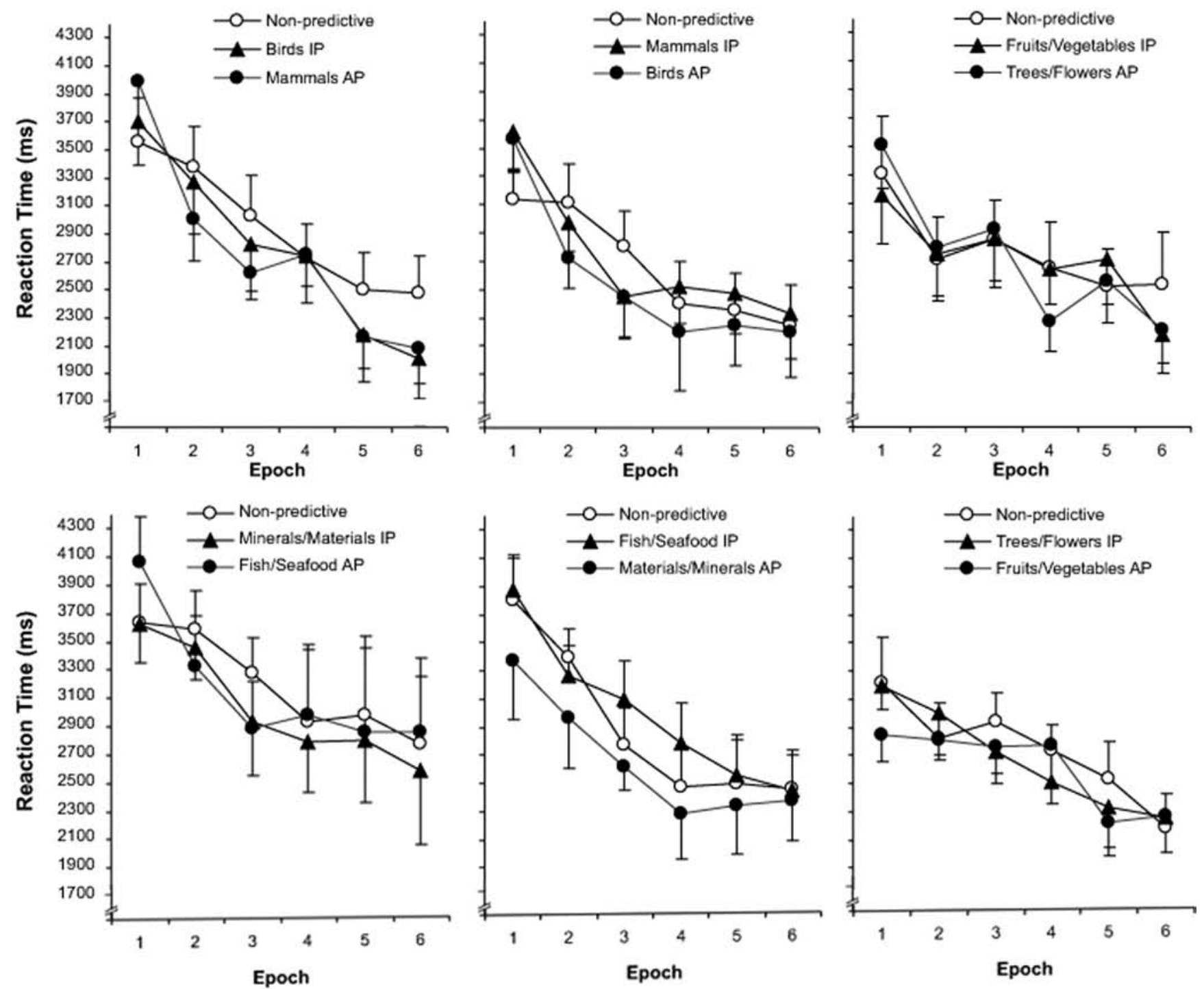

Figure C3. Results of Experiment 3. Mean target detection reaction time (in milliseconds) in the attended predictive (AP) and ignored predictive (IP) contexts (mammals, birds, trees-flowers, fruits-vegetables, fishseafood, and materials-minerals) and in their matched nonpredictive conditions. The error bars represent the standard error of the mean $(N=6)$. 

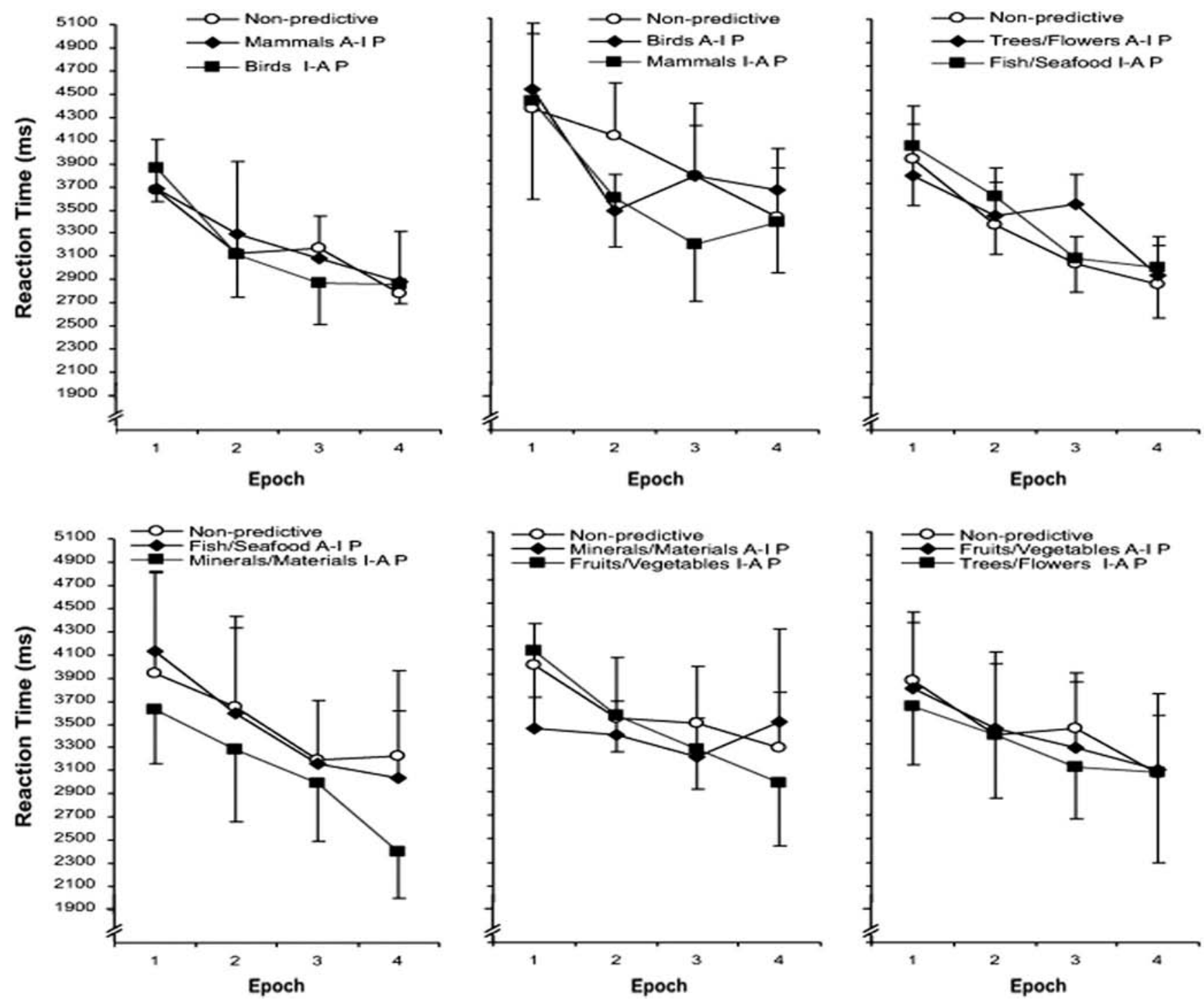

Figure C4. Results of Experiment 4. Mean target detection reaction time (in milliseconds) in the attended-thenignored predictive (A-I P) and ignored-then-attended predictive (I-A P) contexts (mammals, birds, trees-flowers, fruits-vegetables, fish-seafood, and materials-minerals) and in their matched nonpredictive conditions. The error bars represent the standard error of the mean $(N=6)$. 
Appendix D

Filler Context Words Used in Experiments 3 and 4

\begin{tabular}{ll}
\hline Office supplies \\
\hline CRAIE \\
ATLAS \\
STYLO \\
CARNET \\
CAHIER \\
FEUTRE \\
TIMBRE \\
CUTTER \\
CISEAUX \\
TROUSSE \\
CROQUIS \\
DOSSIER \\
PINCEAU \\
PLUME \\
GOMME \\
COLLE \\
AGENDA \\
MANUEL \\
DESSIN \\
CRAYON \\
TAMPON \\
PALETTE \\
CALEPIN \\
COLLAGE \\
TABLEAU \\
ARCHIVE \\
\end{tabular}

Received March 5, 2007

Revision received November 22, 2007

Accepted November 26, 2007 